\title{
Optimal Spread Spectrum Watermark Embedding via a Multistep Feasibility Formulation
}

\author{
H. Oktay Altun, Student Member, IEEE, Adem Orsdemir, Student Member, IEEE, \\ Gaurav Sharma, Senior Member, IEEE, and Mark F. Bocko, Member, IEEE
}

\begin{abstract}
We consider optimal formulations of spread spectrum watermark embedding where the common requirements of watermarking, such as perceptual closeness of the watermarked image to the cover and detectability of the watermark in the presence of noise and compression, are posed as constraints while one metric pertaining to these requirements is optimized. We propose an algorithmic framework for solving these optimal embedding problems via a multistep feasibility approach that combines projections onto convex sets (POCS) based feasibility watermarking with a bisection parameter search for determining the optimum value of the objective function and the optimum watermarked image. The framework is general and can handle optimal watermark embedding problems with convex and quasi-convex formulations of watermark requirements with assured convergence to the global optimum. The proposed scheme is a natural extension of set-theoretic watermark design and provides a link between convex feasibility and optimization formulations for watermark embedding. We demonstrate a number of optimal watermark embeddings in the proposed framework corresponding to maximal robustness to additive noise, maximal robustness to compression, minimal frequency weighted perceptual distortion, and minimal watermark texture visibility. Experimental results demonstrate that the framework is effective in optimizing the desired characteristic while meeting the constraints. The results also highlight both anticipated and unanticipated competition between the common requirements for watermark embedding.
\end{abstract}

Index Terms-Optimum watermark design, projections onto convex sets (POCS), set theoretic watermarking, spread spectrum watermarking.

\section{INTRODUCTION}

D IGITAL watermarking is a special form of digital communication where a multimedia cover signal is utilized as the carrier of auxiliary information. This carrier data, along with

Manuscript received January 18, 2008; revised September 28, 2008. Current version published January 09, 2009. Part of this work was presented at ICIP 2006. This work was supported by the Air Force Office of Scientific Research (AFOSR) under grant number FA9550-07-1-0017. The associate editor coordinating the review of this manuscript and approving it for publication was Prof. Dan Schonfeld.

H. O. Altun, A. Orsdemir, and M. F. Bocko are with the Electrical and Computer Engineering Department, University of Rochester, Rochester, NY 14627-0126 USA (e-mail: altun@ece.rochester.edu; orsdemir@ece.rochester.edu; bocko@ece.rochester.edu).

G. Sharma is with the Electrical and Computer Engineering Department, University of Rochester, Rochester, NY 14627-0126 USA, and also with the Department of Biostatistics and Computational Biology, University of Rochester Medical Center, Rochester, NY 14642 USA (e-mail: gaurav.sharma@ rochester. edu).

This paper has supplementary PDF material available at http://ieeexplore. ieee.org, provided by the authors. This material is $2.7 \mathrm{MB}$ in file size. The supplementary material contains additional images that demonstrate the performance of the optimum watermarking techniques proposed in this paper.

Color versions of one or more of the figures in this paper are available online at http://ieeexplore.ieee.org.

Digital Object Identifier 10.1109/TIP.2008.2008222 the embedded watermark, is communicated to the receiver over a channel that may include distortion and noise. The receiver extracts the watermark information for its use, possibly using a cryptographic key shared with the embedder in the extraction process. Watermarking is often the only solution available when secure out-band channels are unavailable for carrying the auxiliary information or when the information is susceptible to removal. Today, watermarking is employed in many areas [2]-[4], including, but not limited to, copyright enforcement [5], authentication [6], broadcast monitoring [7], and fingerprinting [8]

The watermark design process is composed of three major steps: coding the watermark data, modulating the coded data with a suitable technique, and embedding the modulated data into the cover file while taking into account the perceptual impact of the embedding and potential interference from the cover and the channel. For instance, conventionally in spread spectrum (SS) embedding [9], if the data to be embedded contains redundancy it may be compressed to reduce the data load, then utilizing a key, a pseudo-noise (pn)-sequence is generated for each bit/symbol to be embedded, and these pn-sequences are then embedded into the cover after perceptual shaping. These steps can be performed separately or jointly, and some of them may be entirely skipped depending on the needs of the application.

The coding step is used to make the watermark data resilient to perturbations introduced during transmission of the watermarked image to the intended users (i.e., error correcting coding), to reduce the size of the watermark data when there is redundancy (i.e., compression), or to provide the necessary adaptation of the information to the cover (i.e., informed coding). Error-coding adds carefully designed redundancy to the watermark data [10]. Compression, on the other hand, works in the opposite direction removing the redundancy of the watermark data thereby making it more fragile. Coding can also be utilized to provide adaptation to the cover, Trellis codes [11] and wet paper codes [12] fall in this class. The modulation step consists of selecting a carrier signal format suitable for carrying the watermark information within the cover. This choice is usually made according to the distortion, capacity, and robustness properties desired of the watermark. The most common forms of modulations are quantization index modulation (QIM) [13] or SS [9] modulation, either of which may be utilized in different domains (e.g., spatial/frequency or wavelet). The watermark embedding step is the final step to adaptively incorporate the modulated watermark signal within the cover signal. This step involves the choice of additive or multiplicative insertion of the data, perceptual shaping of the 
carrier signal, and the interference management between the cover and the watermark signals.

In this paper we focus on the watermark embedding step, specifically for zero-bit spread spectrum watermarking. Embedding techniques can be classified as optimization formulations, feasibility formulations, and heuristic methodologies. Heuristic methods are usually practical, computationally efficient, and intuitive ways of embedding watermarks into cover files. These methods dominate the literature and early versions of the conventional modulation methods fall into this category [9], [13]. The methods usually do not offer systematic guarantees for satisfying all the criteria, however, their simplicity makes them rather useful. In optimization formulations of watermark embedding, the watermark insertion is formulated as a constrained optimization problem [14]-[16]. The requirements for the watermarked signal are expressed in analytical form as an optimization of one performance metric while meeting requirements for other performance metrics. Various scenarios may be formulated in this framework. For instance, the designer may wish to minimize the visual distortion due to embedding while maintaining a desired level of watermark embedding strength and robustness to compression. In other applications, the maximization of watermark embedding strength or robustness to compression can be the key objective. Feasibility formulations of watermark embedding [17], [18] provides a middle ground between optimization methods and heuristic methods, where a watermark signal meeting desired criteria is determined without explicitly optimizing a performance criterion. Feasibility formulations are typically computationally less demanding compared with the optimization formulations. Specifically, set theoretic watermarking [17] provides a flexible and readily adaptable framework in which a wide variety of constraints can be incorporated.

In this paper, we consider several optimal watermark embedding formulations for a zero-bit spread spectrum watermarking problem, where the detector is only concerned with establishing the presence/absence of the watermark. We demonstrate that these different formulations can be addressed in a common framework that combines multistep feasibility based optimization [19] with projections onto convex sets (POCS) based set theoretic watermarking [17] for solving the feasible watermarking problem at each step. The method of POCS has been successfully applied to a number of different image processing problems [20]-[25]. Our work can be viewed in this context as an extension of POCS that addresses the multiple requirements inherent in watermarking applications while simultaneously allowing for optimization of one chosen attribute. The resulting methodology for optimal watermark embedding is general and allows a number of convex optimization criteria under convex constraints.

\section{OPTIMAL WATERMARK EMBEDDING}

Depending on the needs of the specific application, some performance requirements of watermarking can be relatively more important compared to others. In medical and military applications, the visual quality is critical since sensitive visual information is communicated. On the other hand, the resilience against compression is more important in network broadcast applications or in other applications when the image is likely to be compressed during transmission. In other applications, watermark detectability with high probability may be desired despite being subject to noise in transmission. In order to emphasize the application specific critical requirement, watermark embedding can be formulated as a constrained optimization formulation where an objective function corresponding to the critical requirement is optimized subject to other constraints. In this paper we will consider various optimal watermark embedding formulations for SS modulation.

SS modulation is a common watermark modulation technique. This type of modulation is widely used in communications due to its wide-band nature and its immunity against narrow-band interference signals and has been successfully adapted to the watermarking field [9]. A key dependent pseudo-noise (pn) sequence $W^{*}$ is inserted ${ }^{1}$ onto the cover image $X_{0}^{*}$ and the detection is performed by correlating the test image $X^{*}$ against the same pn-sequence. Among the many available correlation metrics, we use a mean corrected linear correlation metric [26, p. 127], in our description. Let $X^{*} \in R^{M \times N}$ denote the image with dimensions $M \times N$ and $W^{*} \in R^{M \times N}$ denote the pn-sequence. We stack the columns of the matrices together to generate vectors $X=\operatorname{vec}\left(X^{*}\right) \in R^{M N}, X_{0}=\operatorname{vec}\left(X_{0}^{*}\right) \in R^{M N}$, and $W_{0}=\operatorname{vec}\left(W^{*}\right) \in R^{M N}$. Throughout the paper we will use this 1-D vector notation and assume that any image operators are also represented as matrices conforming to the vector representation. The mean corrected correlation is represented as $W^{T}(X-\bar{X})$ where $W=W_{0}-\overline{W_{0}}$, and $\bar{V}$ denotes the sample mean of the entries in the vector $V$. The presence/absence of the watermark is determined by comparing the estimated correlation value against a threshold $\gamma_{d}$, i.e., if $W^{T}(X-\bar{X}) \geq \gamma_{d}$ the watermark is deemed present and if $W^{T}(X-\bar{X})<\gamma_{d}$ the watermark is deemed to be absent from the image. ${ }^{2}$ We consider next a specific optimal watermark embedding formulation for SS watermarking. Using the notation and terminology established in that context we subsequently define three other optimal watermark embedding formulations for different criteria.

\section{A. Maximization of Embedding Strength}

In applications where maximal robustness against additive noise is required, it is desirable that the watermarked image at the embedder maximize the mean corrected correlation $W^{T}(X-\bar{X})$, which we refer to as watermark embedding strength. The maximization of the embedding strength must clearly be subject to other constraints. The distortion in the watermarked image with respect to (w.r.t.) the original image $X_{0}$ should be perceptually tolerable. In addition, in some applications, it may be desirable that the watermark survive compression up to a given quality level. In order to account for these constraints, we formulate the optimal watermark

\footnotetext{
${ }^{1}$ Our formulation requires only a definition of the detector. The embedding is an implicit outcome of the optimization.

${ }^{2}$ Note that we assume oblivious detection, where the cover is unavailable at the detector. The nonoblivious case may be similarly formulated and actually represents a simplification.
} 
embedding for maximization of embedding strength as the constrained optimization

$$
\begin{aligned}
\max _{X} & W^{T}(X-\bar{X}) \\
\text { subject to } & \left\|H X-H X_{0}\right\| \leq \theta \\
& D_{L}\left(X_{0}\right) \leq\left(X-X_{0}\right) \leq D_{U}\left(X_{0}\right) \\
& W^{T}\left(\mathcal{T}_{\mathcal{I}}\left(Q\left[\mathcal{T}_{\mathcal{F}}(X)\right]\right)-\overline{\mathcal{T}_{\mathcal{I}}\left(Q\left[\mathcal{T}_{\mathcal{F}}(X)\right]\right)}\right) \geq \gamma_{c}
\end{aligned}
$$

where the first two constraints (1) and (2) capture the requirements of perceptually tolerable distortion and the third represents the compression resilience requirement. These constraints and the associated symbols in their definitions are defined next.

Constraint (1) is based on the contrast sensitivity of the human visual system (HVS), where $H$ is a linear spatial filter that represents the characteristics of the HVS. Specifically, the higher sensitivity of the HVS to lower frequencies compared with higher frequencies is encapsulated in $H$. The term $\left\|H X-H X_{0}\right\|$ thus represents a frequency weighted measure representing the perceptual distortion in the candidate watermark image $X$. In the present work, we use the parametric contrast sensitivity model proposed by Mannos and Sakrison [27]. For the model, the spatial filter $H$ is represented in the frequency domain by a radially isotropic function $H\left(f_{r}\right)=2.6\left[0.0192+0.114 f_{r}\right] e^{-\left(0.114 f_{r}\right)^{1.1}}$, where $f_{r}$ denotes the radial frequency in cycles per degree [27].

Constraint (2) exploits texture masking by virtue of which watermark distortion introduced in textured regions is less visible than in smooth regions. The $D_{L}\left(X_{0}\right)$ and $D_{U}\left(X_{0}\right)$ in (2) represent pixel-wise upper and lower bounds on tolerable distortion that are determined from the cover image $X_{0}$. For determining $D_{L}\left(X_{0}\right)$ and $D_{U}\left(X_{0}\right)$, we use the model of Pereira et al. [14], which is briefly summarized in Appendix I. Based on the development in Appendix I, we will find it notationally convenient, in the ensuing presentation, to represent constraint (2) in an alternate form as

$$
\left\|D_{0}\left(X-X_{0}\right)\right\|_{\infty} \leq s_{0}
$$

where $D_{0}$ is a diagonal matrix determined from the original image $X_{0}$ using the texture masking model and $\|V\|_{\infty}=\max _{j}\left|V_{j}\right|$ represents the $\infty$-norm of the vector $V$. The term $\left\|D_{0}\left(X-X_{0}\right)\right\|_{\infty}$ represents a measure of the (worst case) visibility of the texture noise introduced in the embedding and the parameter $s_{0}$ represents a bound on this measure.

Constraint (3) represents the compression resilience requirement for transform domain compression, where $\mathcal{T}_{\mathcal{F}}$ represents the forward transform, $Q[$ ] represents the quantization operation in the transform domain. $\mathcal{T}_{\mathcal{I}}$ represents the inverse transform. The constraint thus enforces a mean corrected correlation greater than $\gamma_{c}$ in the compressed image. Specifically, in this paper we consider JPEG compression, where $\mathcal{T}_{\mathcal{F}}$ and $\mathcal{T}_{\mathcal{I}}$ represents the forward and inverse discrete cosine transforms (DCTs), respectively, and $Q[]$ is the JPEG quantization function at a predetermined quality level. In the transform coding [28], the quantizer $Q[]$ in fact consist of $M N$ distinct scalar quantizers $\left\{Q^{k}[]\right\}_{k=0}^{M N-1}$ for each of the $M N$ transform domain coefficients. Thus, for JPEG compression the term $k$ indexes the 64 different quantizers used for $8 \times 8$ block DCT coefficients, repeatedly tiled so as to cover the entire image. The formulation addresses any transform domain coding scenarios where the transform domain coefficients are quantized via scalar quantizers.

\section{B. Minimization of Frequency Weighted Perceptual Distortion}

For applications, where a minimization of the frequency weighted perceptual distortion metric is desirable, the optimal watermark embedding problem can be formulated as

$$
\begin{aligned}
\min _{X} & \left\|H X-H X_{0}\right\| \\
\text { subject to } & D_{L}\left(X_{0}\right) \leq\left(X-X_{0}\right) \leq D_{U}\left(X_{0}\right) \\
& W^{T}(X-\bar{X}) \geq \gamma_{e} \\
& W^{T}\left(\mathcal{T}_{\mathcal{I}}\left(Q\left[\mathcal{T}_{\mathcal{F}}(X)\right]\right)-\overline{\mathcal{T}_{\mathcal{I}}\left(Q\left[\mathcal{T}_{\mathcal{F}}(X)\right]\right)}\right) \geq \gamma_{c}
\end{aligned}
$$

where $\gamma_{e}$ denotes the desired embedding strength at the embedder, and all the other terms are as defined earlier. Normally $\gamma_{e}$ is chosen to be larger than the detection threshold $\gamma_{d}$ at the watermark detector. The gap $\left(\gamma_{e}-\gamma_{d}\right)$ characterizes the robustness of the watermark and can be related to the probability of error for watermark detection following postwatermarking perturbations [2].

\section{Maximization of Robustness to Compression}

For scenarios where maximal compression resilience is desirable, an alternate optimal watermark embedding problem can be formulated as

$$
\begin{aligned}
\max _{X} & W^{T}\left(\mathcal{T}_{\mathcal{I}}\left(Q\left[\mathcal{T}_{\mathcal{F}}(X)\right]\right)-\overline{\mathcal{T}_{\mathcal{I}}\left(Q\left[\mathcal{T}_{\mathcal{F}}(X)\right]\right)}\right) \\
\text { subject to } & \left\|H X-H X_{0}\right\| \leq \theta \\
& D_{L}\left(X_{0}\right) \leq\left(X-X_{0}\right) \leq D_{U}\left(X_{0}\right) \\
& W^{T}(X-\bar{X}) \geq \gamma_{e} .
\end{aligned}
$$

\section{Minimizing Watermark Texture Visibility}

In a manner analogous to the preceding formulations, one may also formulate the optimal embedding problem to minimize a metric representing the perceptibility of the texture noise introduced by watermark embedding. The problem may be mathematically formulated as

$$
\begin{aligned}
\min _{X} & \left\|D_{0}\left(X-X_{0}\right)\right\|_{\infty} \\
\text { subject to } & \left\|H X-H X_{0}\right\| \leq \theta \\
& W^{T}\left(\mathcal{T}_{\mathcal{I}}\left(Q\left[\mathcal{T}_{\mathcal{F}}(X)\right]\right)-\overline{\mathcal{T}_{\mathcal{I}}\left(Q\left[\mathcal{I}_{\mathcal{F}}(X)\right]\right)}\right) \geq \gamma_{c} \\
& W^{T}(X-\bar{X}) \geq \gamma_{e} .
\end{aligned}
$$

As indicated earlier, the development of this metric is presented in Appendix I. 


\section{CONVEX OPTIMIZATION FRAMEWORK FOR OPTIMAL WATERMARK EMBEDDING}

For the optimal watermark embedding formulations in the preceding section to be useful, efficient methods for determining the solution for the corresponding problems are required. We observe that with the exception of compression resilience, other constraints and objective functions in our formulations are inherently convex. Note that the watermark texture perceptibility constraint depends on the cover image in a complex fashion. However, given the cover image it is manifested simply as an upper and lower bound constraint on pixel values that is clearly convex [17], [29]. Since convex optimization problems have unique global optima and there are a variety of well behaved optimization algorithms for the problems, we consider the optimal watermark embedding problem when the objective function and the constraints are convex.

To addresscompression resilience, we introduce the convex (in fact linear) approximation $W^{T}\left(\mathcal{I}_{\mathcal{I}}\left(Q\left[\mathcal{T}_{\mathcal{F}}(X)\right]\right)-\overline{\mathcal{T}_{\mathcal{I}}\left(Q\left[\mathcal{T}_{\mathcal{F}}(X)\right]\right)}\right) \approx$ $W^{T}\left(\mathcal{T}_{\mathcal{I}}\left(Q_{0}\left[\mathcal{T}_{\mathcal{F}}(X)\right]\right)-\overline{\mathcal{T}_{\mathcal{I}}\left(Q_{0}\left[\mathcal{T}_{\mathcal{F}}(X)\right]\right)}\right) \quad$ where $\quad Q_{0}[\quad]$ represents the approximation of $Q[$ ] obtained by approximating the scalar quantizer $Q^{k}[]$ for the $k$ th transform domain coefficient by $Q_{0}^{k}$ [ ] which is defined as

$Q_{0}^{k}[t]= \begin{cases}0, & Q^{k}\left[\left(\mathcal{T}_{\mathcal{F}}\left(X_{0}\right)\right)_{k}\right]=0 \quad k=0,1, \ldots, M N-1 \\ t, & \text { otherwise. }\end{cases}$

This approximates the compression operation as a projection onto the subspace of transform bases that the quantization operation in compression does not obliterate. The efficacy of this approximation for JPEG compression has been explored in [17]. Given the cover image, the concatenation $\mathcal{T}_{\mathcal{C}}(.) \stackrel{\text { def }}{=} \mathcal{T}_{\mathcal{I}}\left(Q_{0}\left[\mathcal{T}_{\mathcal{F}}().\right]\right)$ is also a linear operator, thus $W^{T}\left(\mathcal{T}_{\mathcal{I}}\left(Q_{0}\left[\mathcal{T}_{\mathcal{F}}(X)\right]\right)-\overline{\mathcal{I}_{\mathcal{I}}\left(Q_{0}\left[\mathcal{I}_{\mathcal{F}}(X)\right]\right)}\right) \geq \gamma_{c}$ represents a linear and, therefore, a convex constraint.

We now consider the optimal watermarking problem formulated in Section II-A and describe it in an alternate general form

$$
\begin{aligned}
\min & \phi_{0}(X) \\
\text { subject to } & \phi_{i}(X) \leq \alpha_{i}, \quad i=1,2,3
\end{aligned}
$$

where $\phi_{0}(X)=-W^{T}(X-\bar{X}), \phi_{1}(X)=\| H X-$ $H X_{0}\left\|, \phi_{2}(X)=\right\| D_{0}\left(X-X_{0}\right) \|_{\infty}$, and $\phi_{3}(X)=$ $-W^{T}\left(\mathcal{T}_{\mathcal{C}}(X)-\overline{\mathcal{T}_{\mathcal{C}}(X)}\right)$, where $\alpha_{1}=\theta, \alpha_{2}=s_{0}, \alpha_{3}=-\gamma_{c}$.

This optimization problem now represents a convex optimization problem. Following [19, p.159], we note that a solution to the problem can be obtained via a multistep feasibility technique that we briefly outline.

1) Initialize $j=0$, and set $u_{0}, l_{0}$ such that the problem (9) has no solution for $\tau=l_{0}$ and has a feasible solution for $\tau=u_{0}$

2) $t_{j}=\left(u_{j}+l_{j}\right) / 2, j=j+1, u_{j}=u_{j-1}$ and $l_{j}=l_{j-1}$.

3) Solve feasibility problem (10) for $\tau=t_{j}$.

4) If there exist a feasible solution, set $u_{j}:=t_{j}$, else $l_{j}=t_{j}$.

5) If $\left(u_{j}-l_{j}\right)>\epsilon$ go to (2), else declare the last feasible solution as the optimum and stop.

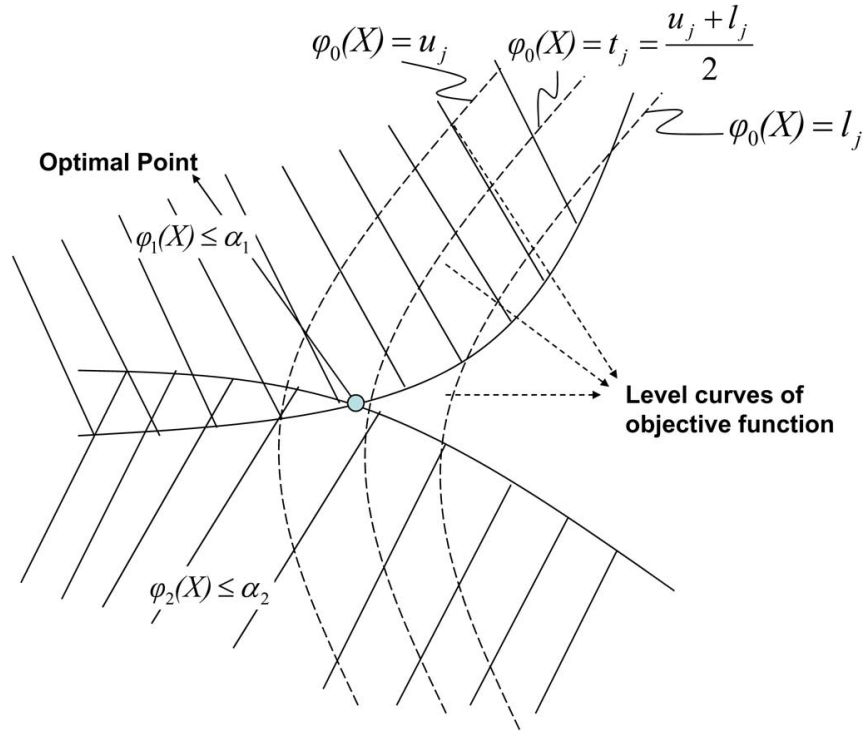

Fig. 1. Bisection search in the multistep feasibility formulation of optimum watermarking.

Let $\tau$ denote a real-valued number, and consider the feasibility problem:

$$
\begin{aligned}
\text { Find } & X \\
\text { subject to } & \phi_{i}(X) \leq \alpha_{i}, \quad i=1,2,3 \\
& \phi_{0}(X) \leq \tau .
\end{aligned}
$$

This feasibility problem is closely related to our optimization problem in (9). In particular if $t^{*}$ is the optimum value for the objective function in our constrained optimization (9), the feasibility problem(10) has a solution if and only if $t_{j} \geq t^{*}$. Suppose next that an algorithm exists for solving the feasibility problem in (10) that provides a feasible $X$ when one exists and indicates that no solution exists otherwise. Then by combining the algorithm with a bisection search on $t_{j}$ we can obtain a solution to the optimization problem as indicated in the aforementioned multistep feasability algorithm. The operation of the algorithm is illustrated pictorially in Fig. 1 for the case of two constraints. At the start of iteration $j, l_{j}<u_{j}$ and the problem (10) has a feasible solution for the upper limit $\tau=u_{j}$ and no feasible solution exists for the lower limit $\tau=l_{j}$. Therefore, $l_{j}<t^{*} \leq u_{j}$. A solution of the feasibility problem is attempted next for the midpoint of the lower and upper limits, i.e., $\tau=t_{j}=l_{j}+u_{j} / 2$. If a solution exists $l_{j}<t^{*} \leq t_{j}$ otherwise $t_{j}<t^{*} \leq u_{j}$. Accordingly, in the former case $u_{j+1}$ is set to $t_{j}$ and in the latter case $l_{j+1}$ is set to $t_{j}$. At the start of the iteration $(j+1)$ once again $l_{j+1}<t^{*} \leq u_{j+1}$ and the process is repeated. In each iteration, the interval $\left[l_{j}, u_{j}\right]$ is bisected so that after exactly $\left[\log _{2}\left(\left(u_{0}-l_{0}\right) / \epsilon\right)\right]$ iterations, the algorithm will terminate, where $\epsilon$ is the tolerance parameter.

\section{A. Multistep Convex Feasibility Formulation of Watermarking}

Since the level set $\left\{X \mid \phi_{0}(X) \leq \tau\right\}$ of the convex objective function $\phi_{0}(\cdot)$ is also a convex set, (10) represents a convex feasibility problem. The solution to this problem can, therefore, be obtained by utilizing the method of POCS [30], 
[31]. Specifically, define the sets $S_{0}(\tau)=\left\{X \mid \phi_{0}(X) \leq \tau\right\}$, and $S_{i}\left(\alpha_{i}\right)=\left\{X \mid \phi_{i}(X) \leq \alpha_{i}\right\}, i=1, \ldots, 3$. Then if $S_{0}(\tau) \bigcap\left(\bigcap_{i=1}^{3} S_{i}\left(\alpha_{i}\right)\right) \neq \emptyset$, starting from any arbitrary initial image $X^{0}$, the sequence of images $\left\{X^{r}\right\}_{r=0}^{\infty}$ converges to a feasible solution of (10), where

$$
X^{r+1}=P_{S_{3}\left(\alpha_{3}\right)}\left(P_{S_{2}\left(\alpha_{2}\right)}\left(P_{S_{1}\left(\alpha_{1}\right)}\left(P_{S_{0}(\tau)}\left(X^{r}\right)\right)\right)\right)
$$

and $P_{S}(Y)=\arg \min _{V \in S}\|Y-V\|$ denotes the projection of $Y$ onto the convex set $S$.

In fact this feasibility problem represents a particular case of the set theoretic watermarking formulation [17]. Projection operators for these sets were enumerated in [17] and are summarized in Appendix II for completeness. Using these projection operators, the feasibility problem (10) can readily be solved using POCS. By employing this "feasibility solver" in the multistep feasibility optimization of Fig. 1, we obtain a solution for the optimal watermark embedding formulation of (10) (which is identical to Section II-A).

For the purpose of concrete illustration, we utilized the optimal watermark embedding problem of Section II-A in our description of the multistep feasibility formulation. One can immediately see that the framework presented here is general and also addresses the optimal embedding formulations of Sections II-B-D (with the convex approximation of compression resilience). Furthermore, additional convex constraints can be readily incorporated in the watermarking process through the addition of suitable constraint sets.

\section{EXPERIMENTAL RESULTS}

We report experimental results for each of the four different optimal watermark embedding scenarios described in Section III. We first outline our experimental setup then we present results for a sample image that highlight the impact of the different formulations. Finally, we present numerical results for the optimizations in tabular format. We note that the watermark detection performance can be inferred in large measure from the constraint parameters and the optimal values. For the feasibility formulation, this has also been extensively investigated in [17]. In this paper, we, therefore, focus our attention on the impact of the different optimizations and the interactions between the optimal values and the constraint parameters.

\section{A. Experimental Setup}

We recall that our cover image has $M \times N$ pixels so that $X$ and $W$ are $M N \times 1$ vectors and $H$ is a $M N \times M N$ matrix representing the HVS contrast sensitivity function as a linear spatially invariant filter. For our SS watermark $W$, we utilize a randomly generated iid sequence taking values +1 and -1 , equiprobably. For the robustness to compression constraints, the quantizer approximation $Q_{0}[]$ was determined corresponding to a JPEG quality factor of 70 .

1) Normalized Constraint Parameter Values and Objective Functions: In order to allow more intuitive understanding of the numerical values used in the simulations and the numerical results of the optimizations, we introduce normalized versions of our simulation parameters that define the constraints and corresponding normalized versions of the objective functions for our four different optimization formulations. For our description we assume that $X_{w}$ denotes the watermarked image obtained from the optimization formulation under discussion.

Observing that $W^{T} W=M N$ represents the correlation value of the watermark sequence with itself, we normalize the embedding strength and the upper bound for the embedding strength by this value. Thus, for the optimization of Section II-A, we report the optimum values of the embedding strength by recording $\nu_{e}=W^{T}\left(X_{w}-\bar{X}_{w}\right) /(M N)$, and for the other formulations we establish the embedding strength constraint in terms of the corresponding normalized parameter value $\bar{\gamma}_{e}=\gamma_{e} /(M N)$. Specifically, we utilize the values $\bar{\gamma}_{e}=$ 1,3 , and 5 in our experiments.

In an analogous fashion, for the optimization formulation of Section II-C, we report the optimal value in the normalized form $\nu_{c}=\left(\mathcal{T}_{\mathcal{I}}\left(Q_{0}\left[\mathcal{T}_{\mathcal{F}}\left(X_{w}\right)\right]\right)-\overline{\mathcal{T}_{\mathcal{I}}\left(Q_{0}\left[\mathcal{T}_{\mathcal{F}}\left(X_{w}\right)\right]\right)}\right) /(M N)$ and for the other formulations the upper-bound parameter for the robustness to compression constraints is given in terms of $\bar{\gamma}_{c}=\gamma_{c} /(M N)$. We use the values $\bar{\gamma}_{c}=1,2$, and 3 in our experiments.

If the image representation has a maximal value of $x_{\max }$ (e.g., $x_{\max }=255$ for 8 bit images), the peak signal energy at the output of the HVS filter $H$ for any input original image can be upper-bounded as

$$
\max _{X_{0}}\left\|H X_{0}\right\|^{2} \leq\|H\|^{2} \max _{X_{0}}\left\|X_{0}\right\|^{2}=\left|\mathcal{H}\left(f_{m}\right)\right|^{2} x_{\max }^{2} M N
$$

where $\mathcal{H}(\cdot)$ denotes the Fourier transform of the HVS contrast sensitivity filter $H$ and $f_{m}$ denotes the frequency at which the magnitude of this Fourier transform attains a maximum. Using this upper bound for normalization, for the optimization formulation of Section II-B, we report the frequency weighted perceptual distortion metric $\left\|H\left(X_{w}-X_{0}\right)\right\|$ in terms of the peak visual signal to watermark power ratio

$$
\operatorname{PSWR}_{v}=10 \log _{10}\left(\frac{\left|\mathcal{H}\left(f_{m}\right)\right|^{2} x_{\max }^{2} M N}{\left\|H\left(X_{w}-X_{0}\right)\right\|^{2}}\right) .
$$

Correspondingly, for the other optimization frameworks, the upper bound for the frequency weighted perceptual distortion is stated in terms of a corresponding upper bound for the peak visual signal to watermark power ratio

$$
\operatorname{PSWR}_{v}^{b}=10 \log _{10}\left(\frac{\left|\mathcal{H}\left(f_{m}\right)\right|^{2} x_{\max }^{2} M N}{\theta^{2}}\right) .
$$

We utilize the values $\operatorname{PSWR}_{v}^{b}=49.68,51.44$, and 54.45 $\mathrm{dB}$ in our experiments corresponding to $\theta$ values of 120000 , 80000 , and 40000, respectively (with $\left|\mathcal{H}\left(f_{m}\right)\right|=0.81$ ). For the watermarked images, we also report the peak signal to watermark power ratio defined as PSWR = $10 \log _{10}\left(255^{2} /\left\|X_{w}-X_{0}\right\|^{2}\right)$.

The $s_{0}$ parameter for the texture masking model denotes the maximum allowable deviation at any pixel and does not require normalization. We use the values $s_{0}=10,20$, and 30 for the constraints. Note that this deviation is infact allowable only in the limit in extremely busy regions of the cover image (where the noise visibility function tends to 1). In the optimization formulation of Section II-D the optimum value of $\sigma_{0}=$ $\left\|D_{0}\left(X_{w}-X_{0}\right)\right\|_{\infty}$ is reported directly and this represents the 


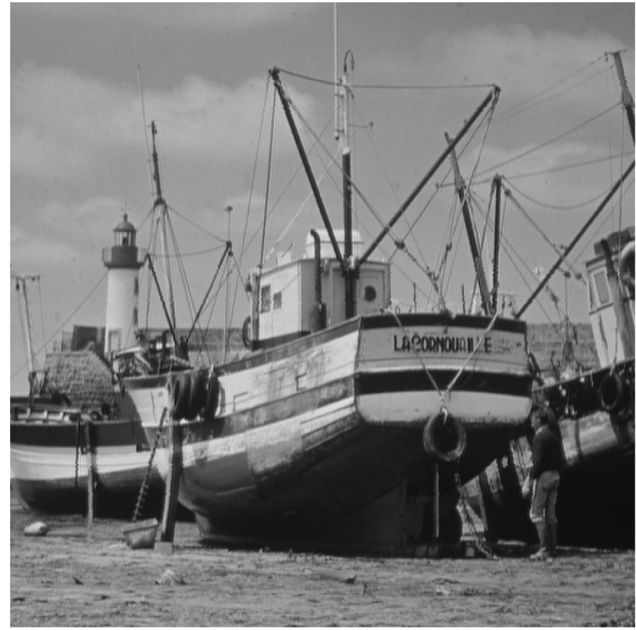

(a)

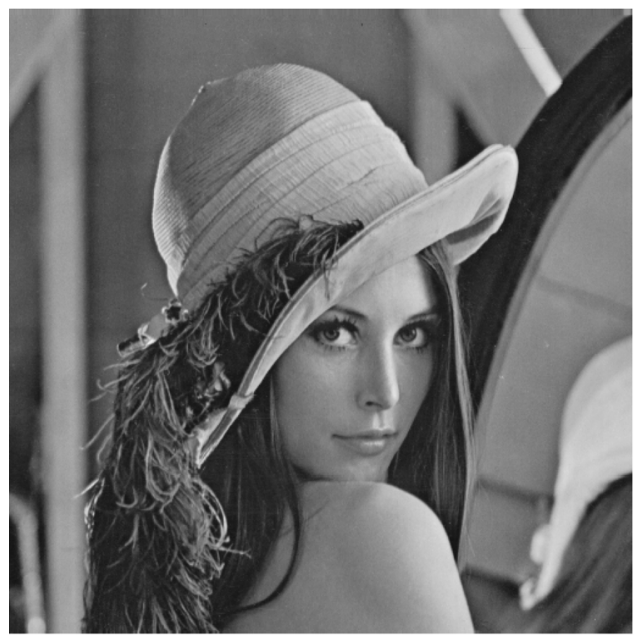

(c)

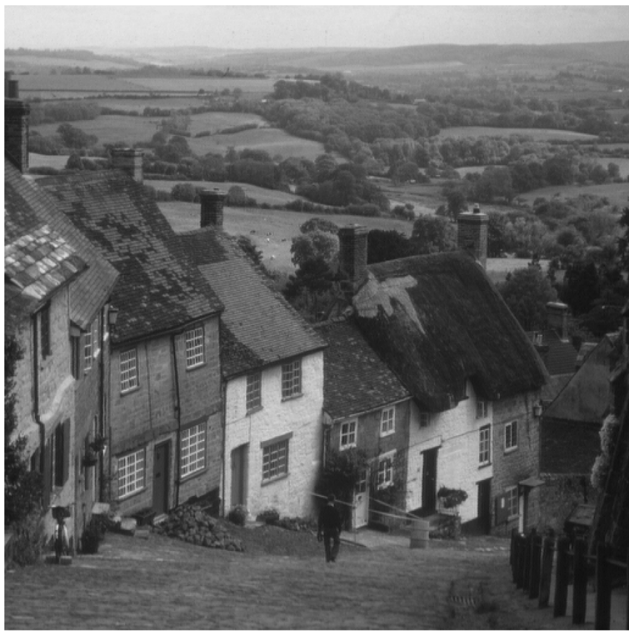

(b)

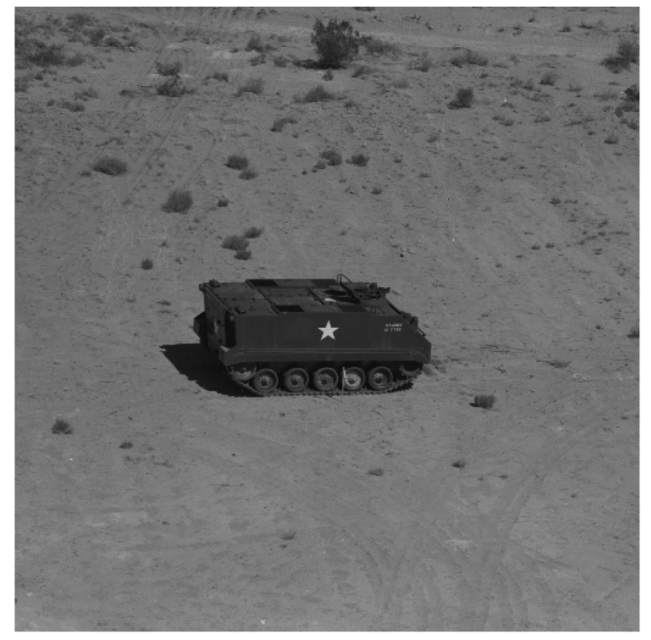

(d)

Fig. 2. Original images. (a) Boat image. (b) Goldhill image. (c) Lena image. (d) Tank image.

maximum noise visibility weighted absolute deviation from the cover image at any pixel location.

2) Test Images: We test our algorithms using four images from USC image database [32], specifically: Lena, Barbara, Tank, and Goldhill. These are 8-bit gray-scale images with a size of $512 \times 512$ pixels. The original versions of these images are shown in Fig. 2. We performed the optimal embedding for different parameter selections for each of the four images for each of the four optimality formulations. In each case, all the different values of the constraint parameters listed in the preceding section were utilized, resulting in 27 different combinations of constraint parameters for each optimization formulation. Due to the computationally intensive nature of the algorithm, our evaluation is limited to this relatively small set of images. Our results, however, serve the desired objective of highlighting the effect of the different optimality formulations and the interaction between the constraints and the optimal values of the objective functions.

\section{B. Watermarked Image for Different Optimal Embeddings}

In order to demonstrate how the choice of the objective function impacts the watermarked image, we begin by presenting results for the Boat image for each of the four optimal embedding formulations. ${ }^{3}$ Since the goal is to highlight differences induced by different objective criteria, constraint parameter values are chosen corresponding to the most relaxed constraints from among the values indicated earlier. This allows the optimization choice over a larger set of possibilities making the impact of the objective function more salient. Specifically, we use $\bar{\gamma}_{e}=1, \bar{\gamma}_{c}=1, \operatorname{PSWR}_{v}^{b}=49.68 \mathrm{~dB}$, and $s_{0}=30$ for defining the required constraints. ${ }^{4}$ Optimal watermarked images corresponding to the four different embedding formulations are shown in Fig. 3. The spatial domain watermark signal $X_{w}-X_{0}$ for each of these optimal embeddings is shown in Fig. 4, where for the purpose of clearer visual presentation, the signal has been scaled by a factor of 4 and translated about a mid gray value of 128 to allow both positive and negative values to be represented. In order to show the distribution of watermark energy in the frequency domain, the magnitude of the discrete Fourier transform (DFT) of the watermark signal $X_{w}-X_{0}$ for each

${ }^{3}$ Corresponding results for the other images are included in supplementary materials posted online.

${ }^{4}$ Note each optimization formulation only requires a subset of three of these parameter values. 


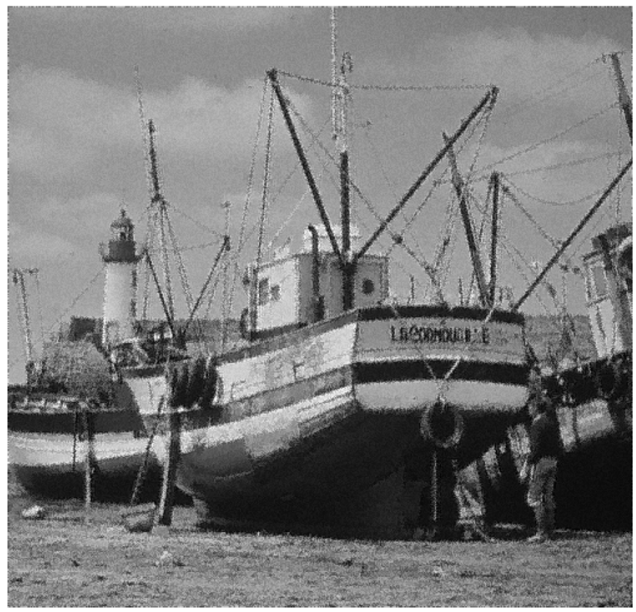

(a)

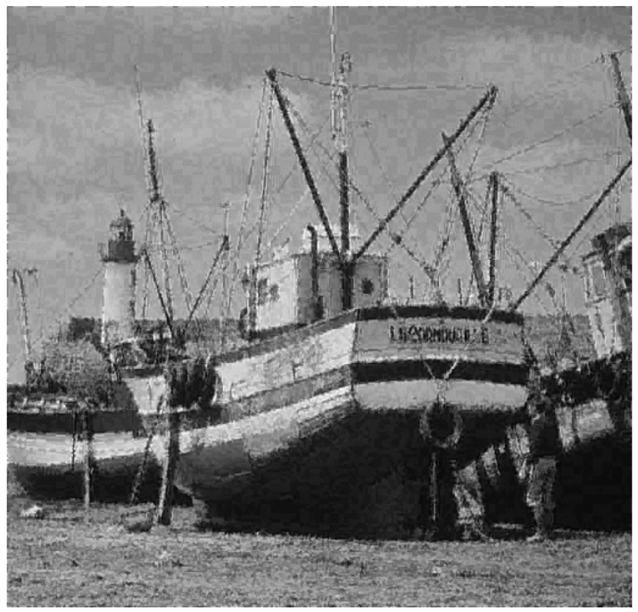

(c)

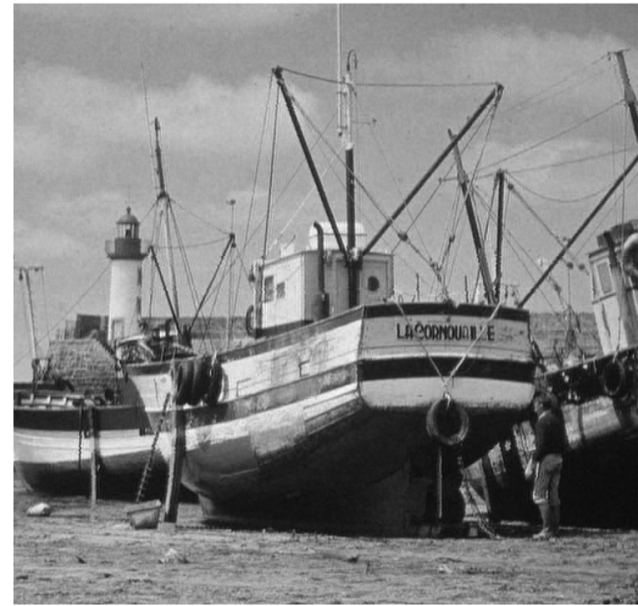

(b)

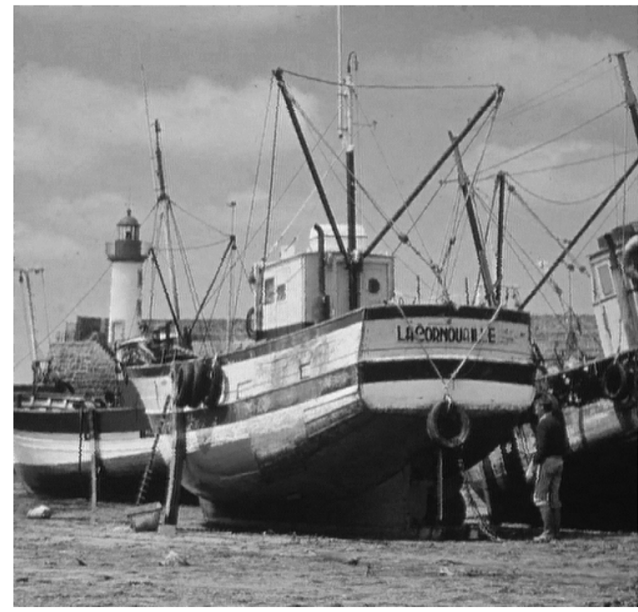

(d)

Fig. 3. Watermarked versions of the Boat image obtained for the four different optimization formulations. (a) Maximizing embedding strength. PSWR of the image is $24.60 \mathrm{~dB}$. (b) Minimizing frequency weighted perceptual distortion. PSWR of the image is $37.71 \mathrm{~dB}$. (c) Maximizing robustness to compression. PSWR of the image is $24.79 \mathrm{~dB}$. (d) Minimizing the watermark texture visibility. PSWR of the image is $37.28 \mathrm{~dB}$.

of these optimal embeddings is shown in Fig. 5. For each of these figures, subfigures (a)-(d) correspond, respectively, to the watermarked images with maximal embedding strength (Section II-A), with minimal frequency weighted perceptual distortion (Section II-B), maximal robustness to compression (Section II-C), and minimal watermark texture noise visibility (Section II-D).

The watermarked Boat image generated by maximizing embedding strength is shown in Fig. 3(a). The PSWR of the watermark image is $24.60 \mathrm{~dB}$. The difference image between watermarked image and the original image, representing the embedded watermark signal is shown in Fig. 4(a) in the spatial domain. This figure clearly shows the adaptation of the embedded watermark signal to the cover image. The textured regions of the image, such as the sandy shore displayed at the bottom of the image, host more watermark power compared to the smoother regions of the image such as the sky region. The effect of the maximization of the detectability algorithm can be observed by examining the frequency domain representation of the watermark in Fig. 5(a). In the frequency domain, despite the watermark power shaping seen in the spatial domain, the designed watermark largely resembles white noise with the power distributed over all the frequency bands. When maximizing the embedding strength the white noise nature of the spread spectrum watermark dominates over the other constraints for this cover image.

The watermarked Boat image generated by minimizing frequency weighted perceptual distortion is shown in Fig. 3(b). The PSWR of the image is $37.71 \mathrm{~dB}$. The watermarked image is visually pleasing. The difference image between watermarked image and the original image in spatial domain is shown in Fig. 4(b). The adaptation of the embedded watermark signal to the cover image is still visible; however, the contrast of the difference image in spatial domain is lower compared to the other algorithms. The effect of the algorithm can be better observed by examining the watermark in frequency domain in Fig. 5(b). The watermark power is pushed out of the visible frequency region. The circular region seen in the middle of the spectrum matches the frequency region over which the filter representing the HVS contrast sensitivity takes on significant values.

The watermarked Boat image generated by maximizing robustness to compression is shown in Fig. 3(c). The PSWR of 


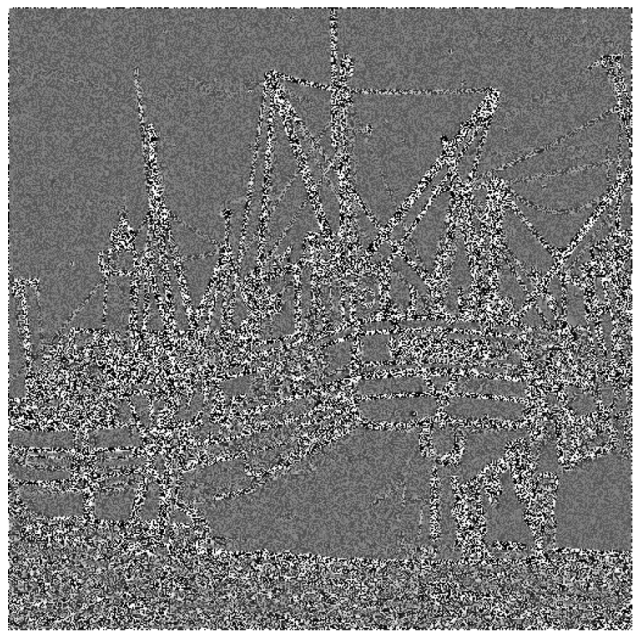

(a)

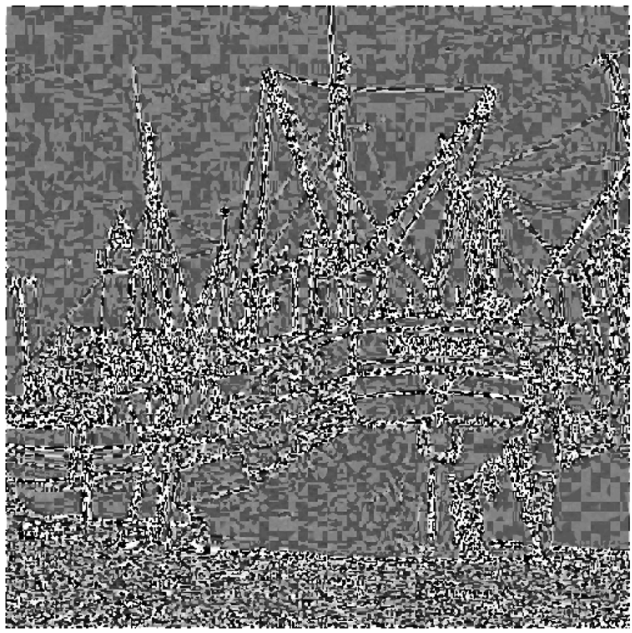

(c)

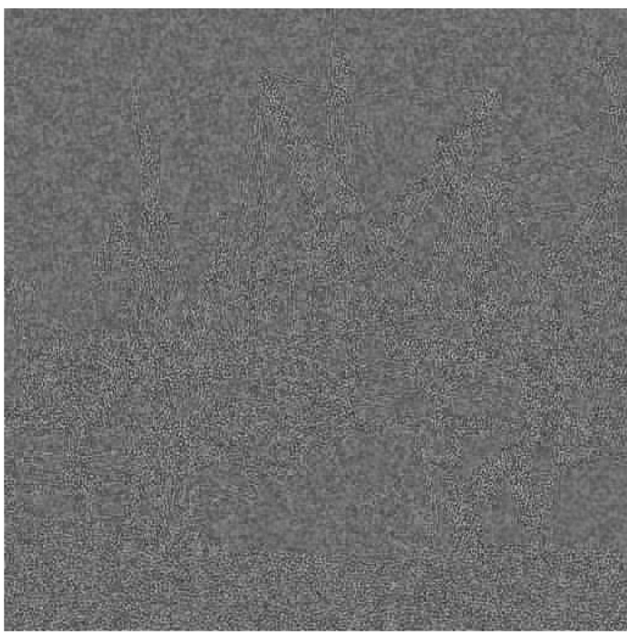

(b)

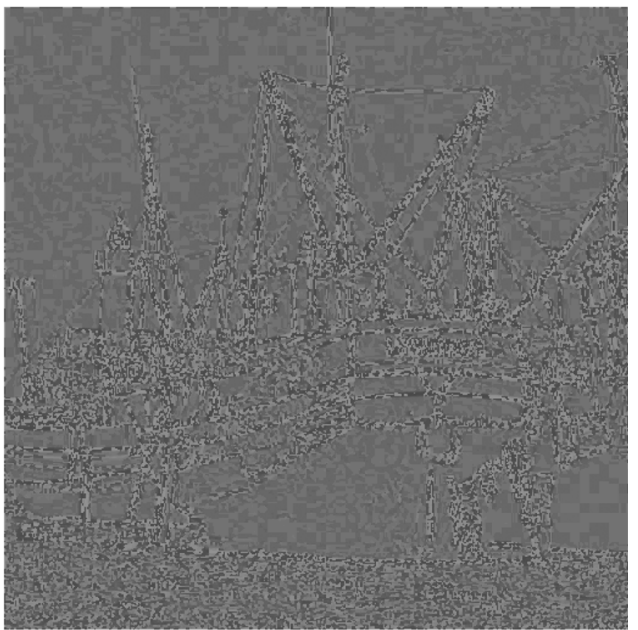

(d)

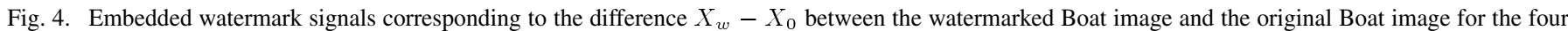

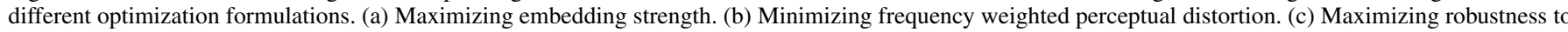

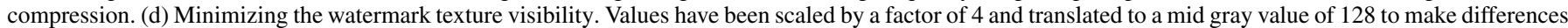
clearer and to allow representation of both positive and negative values.

this image is $24.79 \mathrm{~dB}$. This optimization scenario introduced visible artifacts in the watermark image that are similar to aggressive JPEG compression artifacts. This is mainly due to the nature of the objective function. In order to provide resilience to JPEG compression the maximization of robustness causes the watermark signal power to be distributed in lower frequency regions of the image, which usually falls into the visible regions. It is important to note that these artifacts can be minimized by tightening the visual quality constraints. However, we utilize rather relaxed visual quality constraints in order to demonstrate the dominant distortion encountered in this optimization. The difference image between watermarked image and the original image (i.e., embedded watermark signal) is shown in Fig. 4(c). This figure illustrates the adaptation of the watermark to the cover image. In addition, some blocking is seen in the embedded watermark signal in less textured regions of the image. The robustness to compression metric measures the watermark power distributed in the DCT bases that are preserved in the original image in the compressed representation. The maximization of this metric, therefore, causes the watermark power to be distributed within these bases. The frequency domain representation of the watermark power in Fig. 5(c) illustrates that the maximization of robustness to compression tends to push the watermark power toward the low frequency region.

The watermarked Boat image generated by minimizing the watermark texture visibility metric is shown in Fig. 3(d). The PSWR of the image is $37.28 \mathrm{~dB}$. The watermarked image is visually pleasing. The difference image between watermarked image and the original image is shown in Fig. 4(d). The watermark power in the spatial domain is concentrated in regions of high spatial activity where the embedded watermarked signal is better masked by the content in the cover image. The spatial distribution of energy in the watermark signal resembles the distribution obtained by maximizing robustness to compression in Fig. 5(c), however, the scales are quite different, the overall distortion being much lower in the present case. The similarity in the spatial distribution of watermark energy exists because more of the high frequency DCT bases are preserved in regions with 


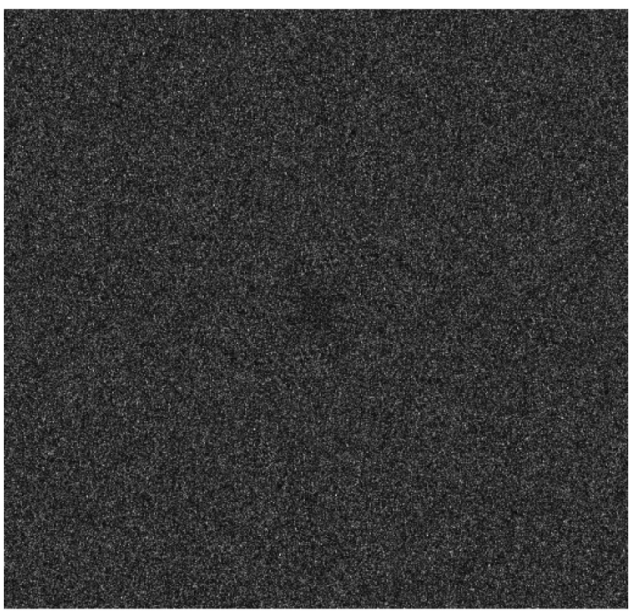

(a)

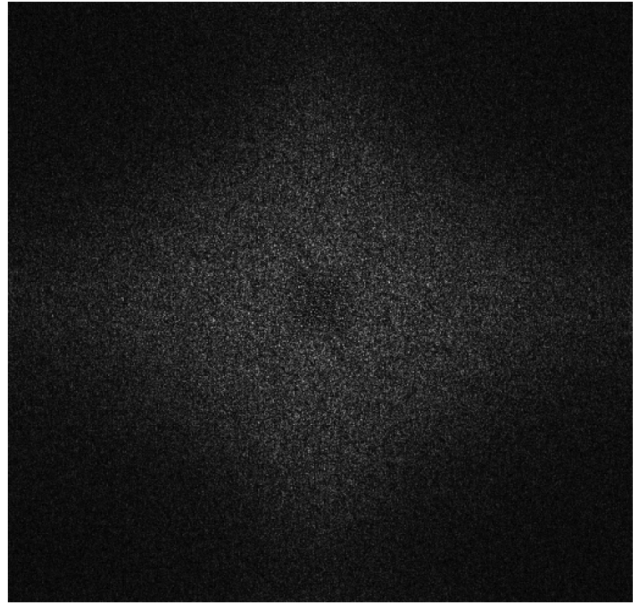

(c)

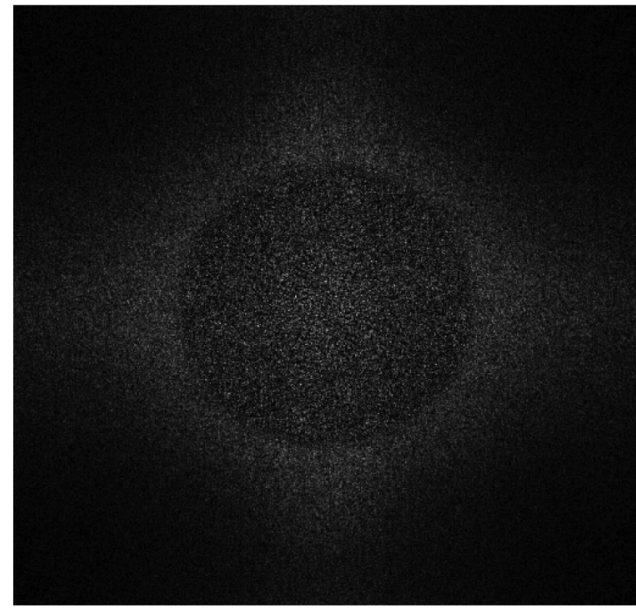

(b)

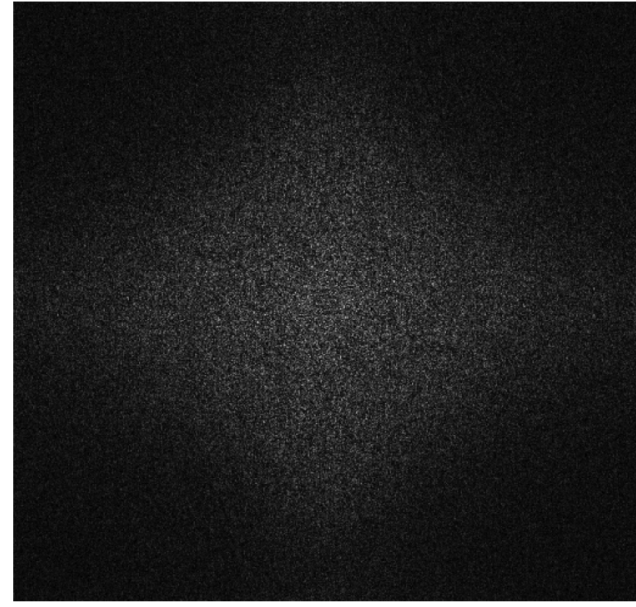

(d)

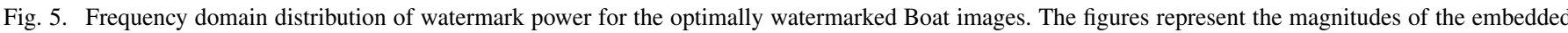

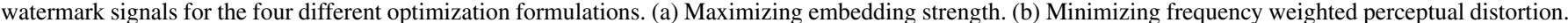
(c) Maximizing robustness to compression. (d) Minimizing the watermark texture visibility.

high spatial activity in the image. The frequency domain representation of the watermark signal in Fig. 5(d) illustrates that the watermark power tends to concentrate at lower frequencies. This is because the spatial domain shaping of the embedded watermark signal also causes the watermark signal power distribution to approximate the low pass characteristic of the cover image.

To illustrate the impact of the cover image content, we present in Figs. 6-8 the watermarked images, the embedded watermark signals in the spatial domain, and the magnitude of the discrete Fourier transforms for the embedded watermark signals for the Lena image for the different optimality formulations. The results largely follow the trends observed for the Boat image, though, of course the shaping of the watermark power adapts to the different content for this image. Specifically comparing Fig. 8(c) and (d) with Fig. 5(c) and (d), respectively, we see that the since the power in the Lena image concentrates in lower frequencies than in the Boat image, so does the watermark signal power for the optimality formulations for maximizing robustness to compression and minimizing watermark texture visibility. The results for the two other images (included in the supplementary materials) support these deductions.

\section{Optimum Values as a Function of Constraint Parameters}

The optimum value of the objective function achievable in each of the constrained optimization formulations depends on the constraint parameters and on the cover image content. In general, changes in parameter values exert a varying influence on the optimum value. We illustrate this by considering each of the optimal formulations and tabulating the optimum values obtained for the objective functions for each of the 4 images for the 27 possible parameter values for the constraints enumerated earlier. The variation in the optimal values with change in constraint parameter values not only indicate the influence of each of the constraint parameters on the optimum but also serve to establish limits for what is attainable under the constraints.

Table I lists the maximum embedding strength $\nu_{e}$ obtained for the formulation of Section II-A for various parameter values. The maximum embedding strength follows expected trends with the change in the constraint parameter values: increasing with an increase in values of $\theta$ and $s_{0}$, and decreasing with an increase in $\bar{\gamma}_{c}$. For some cases, however, the relaxation of a constraint does not change the optimal value because the constraint 


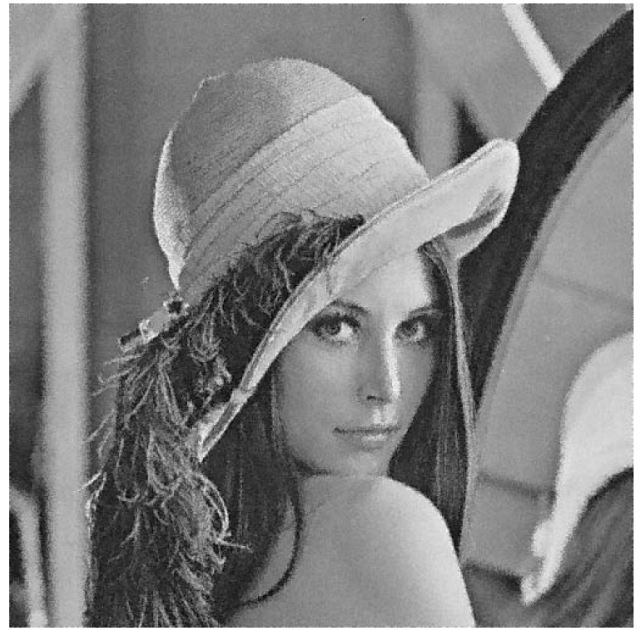

(a)

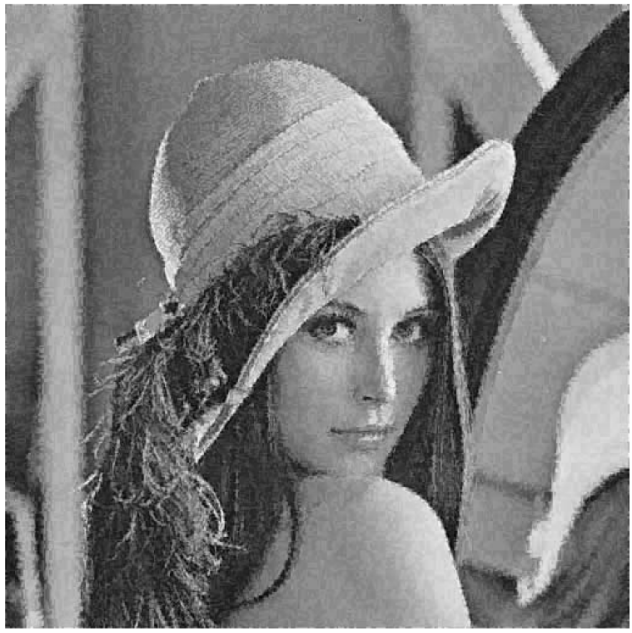

(c)

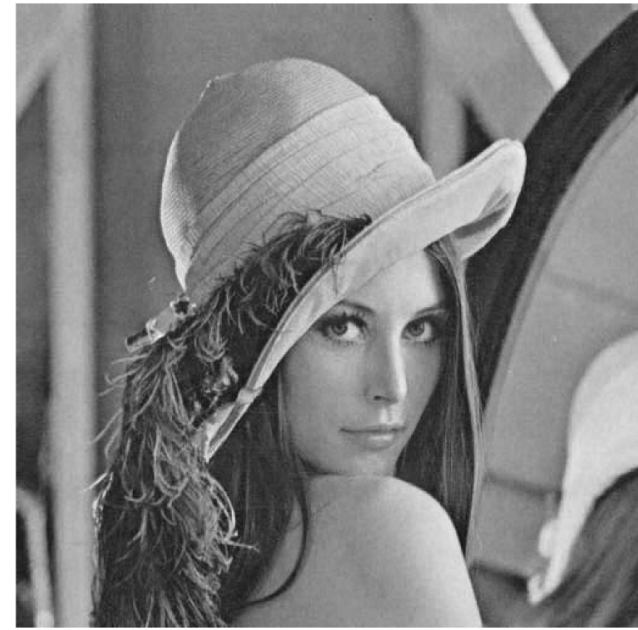

(b)

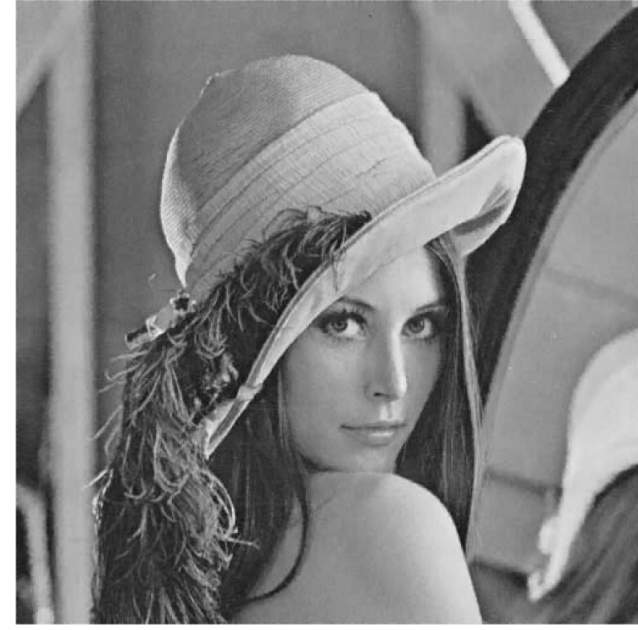

(d)

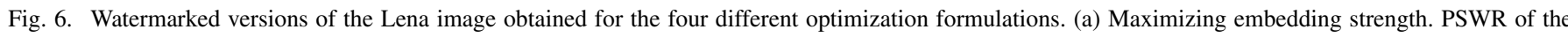

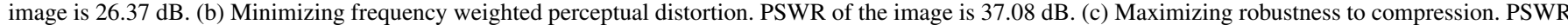
of the image is $26.14 \mathrm{~dB}$. (d) Minimizing the watermark texture visibility. PSWR of the image is $36.71 \mathrm{~dB}$

is inactive at the optima. In general, the watermark texture visibility constraint parameter $s_{0}$ exerts the most influence on the optimal value of the embedding strength, this is followed in decreasing order by the robustness to compression constraint parameter $\bar{\gamma}_{c}$ and by the frequency weighted perceptual distortion bound $\operatorname{PSWR}_{v}^{b}$. The maximal values of $\nu_{e}$ also exhibit variation along expected lines based on the content of the cover image: the maximal values for the same parameter values for the constraints increase progressively as the cover image becomes busier (in the order Lena, Tank, Boat, and Goldhill).

Table II summarizes the minimum frequency weighted distortion obtained for the formulation of Section II-B using the proposed optimization methodology where the optimal values are reported in terms of the peak visual signal to watermark power ratio $\mathrm{PSWR}_{v}$ defined earlier. The peak visual signal to watermark power ratio increases as the constraints are relaxed but once again demonstrates several scenarios where a relaxation of one or two of the constraints does not change the optimal value due to the constraints being nonbinding. The numerical values indicate that the visual texture model constraint parameter $s_{0}$ exerts the strongest influence on the optimal value fol- lowed by the robustness to compression constraint parameter $\bar{\gamma}_{c}$ and then by the embedding strength parameter $\bar{\gamma}_{e}$, in decreasing order of impact. The strong influence of $s_{0}$ reflects the complementary nature of the two visual constraints. Whereas, the maximization of the frequency weighted measure of perceptual distortion $\mathrm{PSWR}_{v}$, which is based on the HVS contrast sensitivity, attempts to move the watermark signal power into high frequencies, it is constrained from moving the power into smooth regions of the image by the watermark texture visibility constraints.

The maximum values for the robustness to compression metric $\nu_{c}$ are enumerated in Table III for the 4 images and each of the 27 values of the constraint parameters. The resulting optimum values of $\nu_{c}$ are higher when the visual constraints are relaxed, i.e., for higher $s_{0}$ and higher $\theta$; however, they diminish with a higher embedding strength constraint, i.e., higher $\bar{\gamma}_{e}$. While this initially appears counter-intuitive, it can be understood from the fact that the robustness to compression and embedding strength compete for the same "perceptual bandwidth" controlled by the visual constraint sets. Among the constraint parameters, the watermark texture visibility 


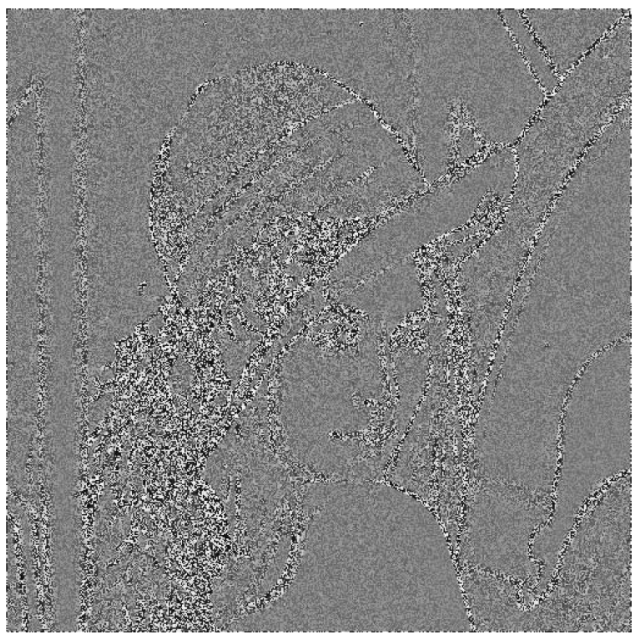

(a)

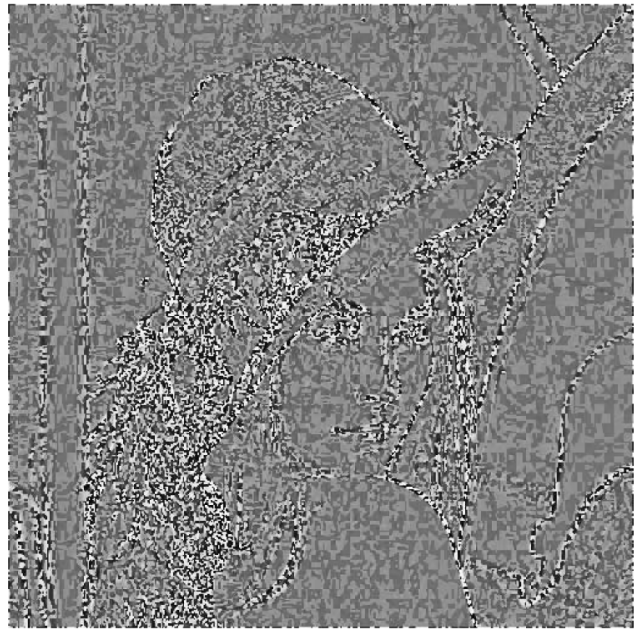

(c)

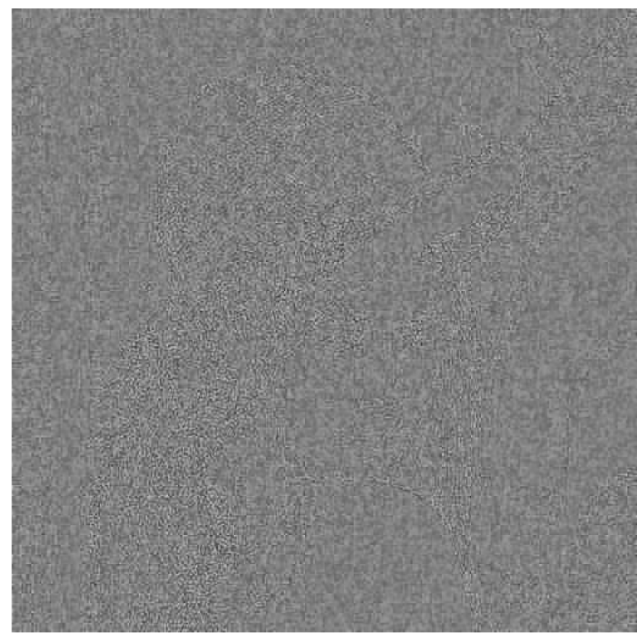

(b)

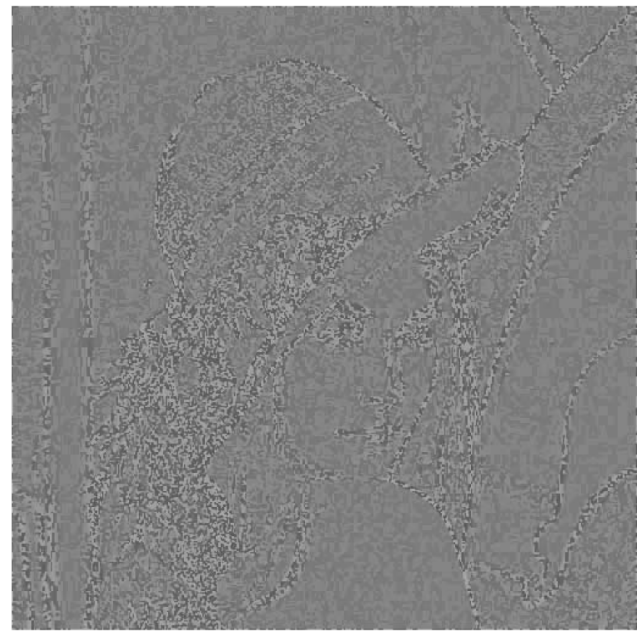

(d)

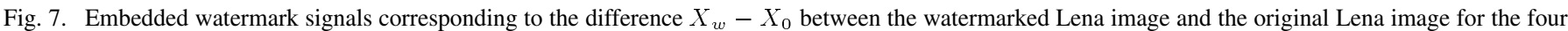

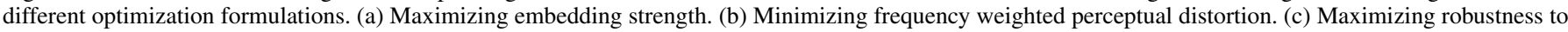

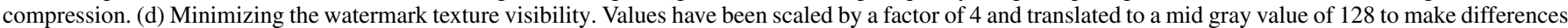
clearer and to allow representation of both positive and negative values.

constraint parameter $s_{0}$ once again exerts the highest influence, followed by the embedding strength parameter $\bar{\gamma}_{e}$ and the frequency weighted perceptual distortion bound $\operatorname{PSNR}_{v}^{b}$, in decreasing order of impact.

Table IV summarizes the minimum values for the texture noise visibility metric $\sigma_{0}=\left\|D_{0}\left(X_{w}-X_{0}\right)\right\|_{\infty}$ obtained for the formulation of Section II-D using the proposed optimization algorithm. The minimum values of $\sigma_{0}$ are nondecreasing as the constraint parameters are adjusted to make the constraints more restrictive. Once again, several cases are seen where a relaxation of one of the constraints does not change the optimum value because the constraint is nonbinding at the optima. In this case, changes in the value of the parameter $\bar{\gamma}_{e}$ corresponding to the embedding strength constraint cause the largest variations in the minimum value of $\sigma_{0}$. The value of $\bar{\gamma}_{c}$, corresponding to the robustness to compression constraint, exercises the next strongest influence on the optimal value, whereas the bound $\operatorname{PSWR}_{v}^{b}$ for the frequency weighted perceptual distortion metric has the smallest influence on the minimal value of $\sigma_{0}$.
The tables and the observations also shed light on the interactions between the different objectives in watermarking applications. In general it is well understood that the objectives of minimizing perceptual distortion and of maximizing embedding strength act in countervailing directions. However, as the results indicate, even the objectives of maximizing embedding strength and of maximizing robustness to compression, which are often thought of as being aligned, can also compete with each other. Likewise, the objectives of minimizing watermark visibility as indicated by texture masking and by the contrast sensitivity function are not entirely aligned. The preceding observations also demonstrate the asymmetric nature of some of the interactions between the different constraints in watermarking: while the watermark texture visibility constraint $s_{0}$ exerts a strong influence on the frequency weighted perceptual distortion metric $\mathrm{PSWR}_{v}$, the converse is not true.

The optimal values listed in Tables I-IV also provide guidance for establishing constraints for watermarking (in optimality and feasibility formulations). When other constraints 


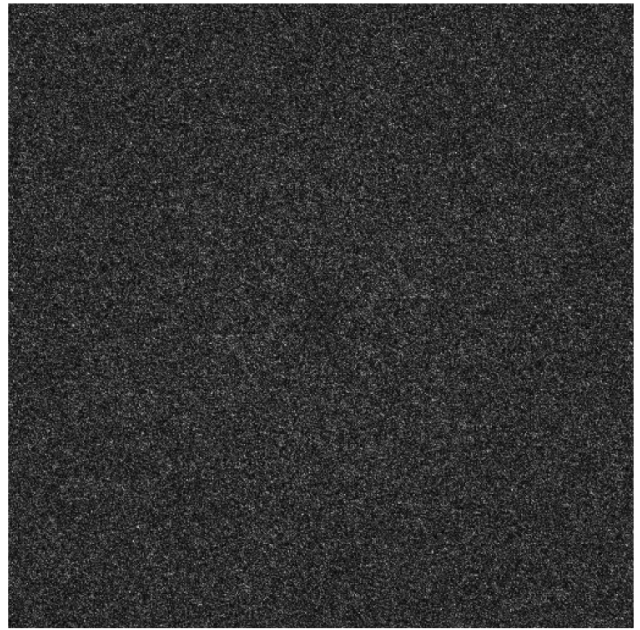

(a)

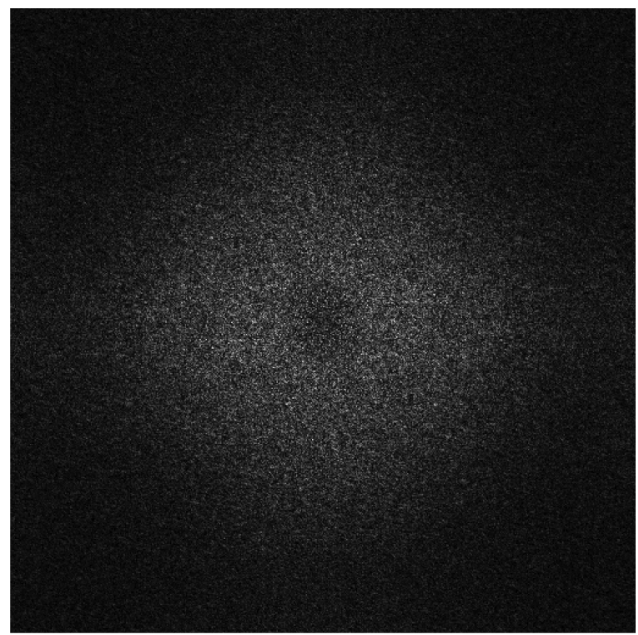

(c)

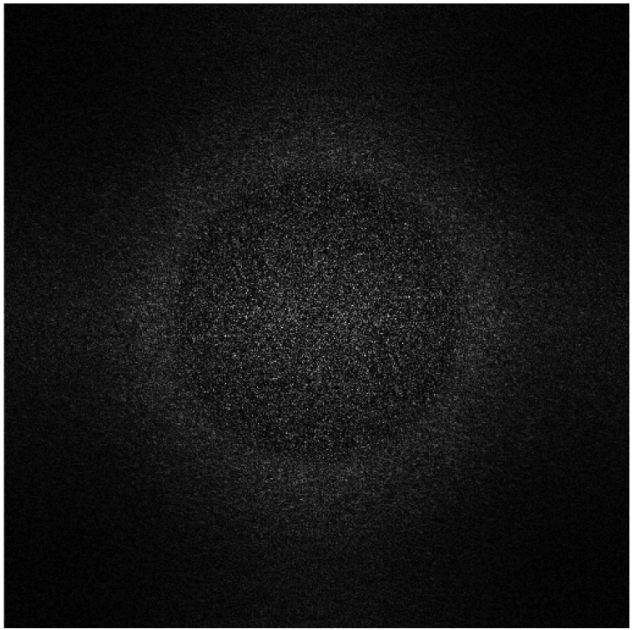

(b)

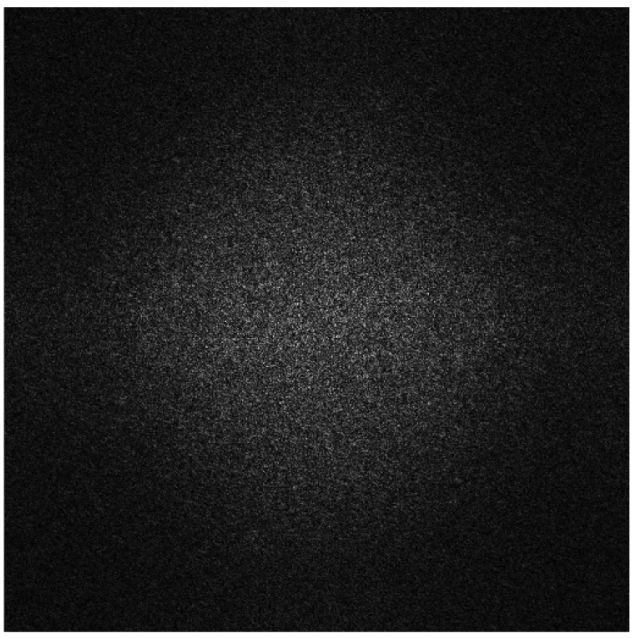

(d)

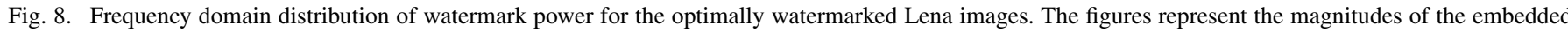

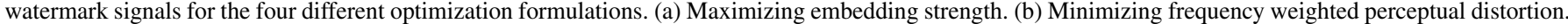
(c) Maximizing robustness to compression. (d) Minimizing the watermark texture visibility.

are kept the same, the optimal value represents the tightest bounds for which a feasible solution exists. Thus, values lower than these upper bounds should be selected when imposing constraints.

\section{Computational Complexity}

The computational burden of the proposed optimization methodology tends to be rather high. An analytic characterization of the computational complexity of the overall method is complicated by the iterative nature of the algorithm with two nested iterations corresponding to the multistep feasibility bi-section and the POCS algorithm for the individual feasibility problems. We, therefore, provide estimates of run time for our sample images for individual steps and for the overall algorithm. All computational times are based on MATLAB implementations under a Windows XP operating system on an AMD 2-GHz Dual Core Athlon 64-bit processor with 2 GB of main memory.

The primitive computational steps of the POCS algorithm for solving the feasibility problems are the projections onto the in- dividual sets. The computational times for the projections onto the individual sets are approximately: $0.050 \mathrm{~s}$ for the projection onto the watermark embedding strength constraint, 0.023 $\mathrm{s}$ for the watermark texture visibility constraint, $5.67 \mathrm{~s}$ for robustness to compression constraint, and $5.90 \mathrm{~s}$ for the frequency weighted perceptual distortion constraint. The nature of the projections accounts for the significant variation in these times. For instance, the projection onto the frequency weighted perceptual distortion set utilizes a (full-frame) discrete Fourier transform of the image, which increases the computational load. One set of successive projections, for all of the constraints requires a computational time of approximately $11.6 \mathrm{~s}$. In our experiments, 100 projections were found to be sufficient to deduce the existence/absence of a feasible solution for each of the optimization frameworks, yielding a worst case estimate of approximately 19 min for solving a feasibility problem. The number of bi-section iterations determines the number of feasibility problems that need to be solved. This is dependent on the values of the upper and lower bounds $u_{0}, l_{0}$ for the optimum value of the optimization in the algorithm of Fig. 1. For instance, for optimization of 
TABLE I

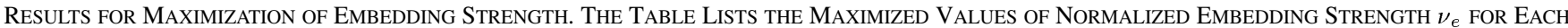

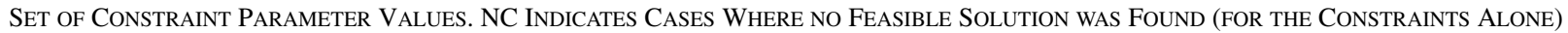

\begin{tabular}{|c|c|c|c|c|c|c|c|c|c|c|}
\hline & & \multicolumn{3}{|c|}{$\bar{\gamma}_{c}=1$} & \multicolumn{3}{|c|}{$\bar{\gamma}_{c}=2$} & \multicolumn{3}{|c|}{$\bar{\gamma}_{c}=3$} \\
\hline & & $s_{0}=10$ & $s_{0}=20$ & $s_{0}=30$ & $s_{0}=10$ & $s_{0}=20$ & $s_{0}=30$ & $s_{0}=10$ & $s_{0}=20$ & $s_{0}=30$ \\
\hline \multirow{3}{*}{ Boat } & $P S W R_{v}^{b}=54.45$ & 3.54 & 7.73 & 11.56 & $\mathrm{NC}$ & 7.34 & 11.56 & $\mathrm{NC}$ & $\mathrm{NC}$ & 11.23 \\
\hline & $P S W R_{v}^{b}=51.44$ & 3.71 & 7.88 & 11.77 & $\mathrm{NC}$ & 7.48 & 11.77 & $\mathrm{NC}$ & $\mathrm{NC}$ & 11.52 \\
\hline & $P S W R_{v}^{b}=49.68$ & 3.71 & 8.06 & 11.93 & $\mathrm{NC}$ & 7.77 & 11.93 & $\mathrm{NC}$ & $\mathrm{NC}$ & 11.63 \\
\hline \multirow{3}{*}{ Goldhill } & $P S W R_{v}^{b}=54.45$ & 3.85 & 7.95 & 11.86 & $\mathrm{NC}$ & 7.86 & 11.86 & $\mathrm{NC}$ & 2.50 & 11.78 \\
\hline & $P S W R_{v}^{b}=51.44$ & 3.97 & 8.11 & 12.09 & $\mathrm{NC}$ & 7.97 & 12.09 & $\mathrm{NC}$ & 5.00 & 12.00 \\
\hline & $P S W R_{v}^{b}=49.68$ & 3.97 & 8.28 & 12.24 & $\mathrm{NC}$ & 8.12 & 12.24 & $\mathrm{NC}$ & 5.00 & 12.11 \\
\hline \multirow{3}{*}{ Lena } & $P S W R_{v}^{b}=54.45$ & 2.18 & 6.62 & 9.83 & $\mathrm{NC}$ & 4.51 & 9.76 & $\mathrm{NC}$ & $\mathrm{NC}$ & 5.00 \\
\hline & $P S W R_{v}^{b}=51.44$ & 2.24 & 6.78 & 9.96 & $\mathrm{NC}$ & 4.92 & 9.91 & $\mathrm{NC}$ & $\mathrm{NC}$ & 7.41 \\
\hline & $P S W R_{v}^{b}=49.68$ & 2.24 & 6.88 & 10.00 & $\mathrm{NC}$ & 4.92 & 9.98 & $\mathrm{NC}$ & $\mathrm{NC}$ & 7.68 \\
\hline \multirow{3}{*}{ Tank } & $P S W R_{v}^{b}=54.45$ & 3.33 & 7.73 & 11.54 & $\mathrm{NC}$ & 6.69 & 11.54 & $\mathrm{NC}$ & $\mathrm{NC}$ & 9.84 \\
\hline & $P S W R_{v}^{b}=51.44$ & 3.35 & 7.89 & 11.74 & $\mathrm{NC}$ & 6.99 & 11.74 & $\mathrm{NC}$ & $\mathrm{NC}$ & 10.00 \\
\hline & $P S W R_{v}^{b}=49.68$ & 3.35 & 8.03 & 11.87 & $\mathrm{NC}$ & 7.01 & 11.87 & $\mathrm{NC}$ & $\mathrm{NC}$ & 10.00 \\
\hline
\end{tabular}

TABLE II

Results for Minimization of Frequency Weighted Perceptual Distortion. The Table Lists the MaXimized Values of NoRmalized PercePtual

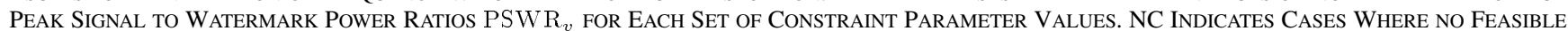
SOLUTION WAS FOUND (FOR THE CONSTRAINTS ALONE)

\begin{tabular}{|c|c|l|l|l|l|l|l|l|l|l|l|}
\hline \multirow{2}{*}{} & & \multicolumn{3}{|c|}{$\bar{\gamma}_{e}=1$} & \multicolumn{3}{|c|}{$\bar{\gamma}_{e}=3$} & \multicolumn{3}{c|}{$\bar{\gamma}_{e}=5$} \\
\cline { 3 - 11 } & & $s_{0}=10$ & $s_{0}=20$ & $s_{0}=30$ & $s_{0}=10$ & $s_{0}=20$ & $s_{0}=30$ & $s_{0}=10$ & $s_{0}=20$ & $s_{0}=30$ \\
\hline \hline \multirow{2}{*}{ Boat } & $\bar{\gamma}_{c}=1$ & 89.47 & 104.66 & 104.66 & 71.54 & 104.66 & 104.66 & $\mathrm{NC}$ & 89.47 & 104.66 \\
& $\bar{\gamma}_{c}=2$ & $\mathrm{NC}$ & 89.47 & 104.66 & $\mathrm{NC}$ & 89.47 & 104.66 & $\mathrm{NC}$ & 74.55 & 104.66 \\
\hline \hline \multirow{5}{*}{ Goldhill } & $\bar{\gamma}_{c}=3$ & $\mathrm{NC}$ & $\mathrm{NC}$ & 89.47 & $\mathrm{NC}$ & $\mathrm{NC}$ & 89.47 & $\mathrm{NC}$ & $\mathrm{NC}$ & 89.47 \\
\hline \hline \multirow{5}{*}{ Lena } & $\bar{\gamma}_{c}=1$ & 89.86 & 105.04 & 105.04 & 77.94 & 105.04 & 105.04 & $\mathrm{NC}$ & 105.04 & 105.04 \\
& $\bar{\gamma}_{c}=3$ & $\mathrm{NC}$ & 89.86 & 105.04 & $\mathrm{NC}$ & 89.86 & 105.04 & $\mathrm{NC}$ & 80.00 & 105.04 \\
& $\bar{\gamma}_{c}=1$ & 61.42 & 103.57 & 103.57 & $\mathrm{NC}$ & 103.57 & 103.57 & $\mathrm{NC}$ & 70.45 & 103.57 \\
\hline \hline \multirow{2}{*}{ Tank } & $\bar{\gamma}_{c}=2$ & $\mathrm{NC}$ & 67.44 & 103.57 & $\mathrm{NC}$ & 64.43 & 103.57 & $\mathrm{NC}$ & $\mathrm{NC}$ & 85.44 \\
& $\bar{\gamma}_{c}=2$ & $\mathrm{NC}$ & $\mathrm{NC}$ & 67.44 & $\mathrm{NC}$ & $\mathrm{NC}$ & 67.44 & $\mathrm{NC}$ & $\mathrm{NC}$ & 55.40 \\
\hline
\end{tabular}

robustness to compression, a choice of upper and lower limits as $l_{0}=0$ and $u_{0}=20$, would require $\left\lceil\log _{2}(20-0) / 0.01\right\rceil=11$ bi-section steps to search for a solution within an $\epsilon=0.01$ neighborhood of the optimal solution. In this case the optimization would take a worst case time of $209 \mathrm{~min}$. In practice, the time required is significantly less than this worst case estimate. Furthermore, utilizing relaxed projections [31] we observed a speed up for the solution of the feasibility problems. In our experiments, the average time for determining an optimal embedding was around $92 \mathrm{~min}$. 
TABLE III

Results for MaXimization of Robustness to Compression. The TABle Lists the MaXimized Values of the NoRmalized Compression

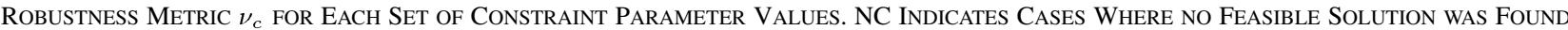
(FOR THE CONSTRAINTS ALONE)

\begin{tabular}{|c|c|c|c|c|c|c|c|c|c|c|}
\hline & & \multicolumn{3}{|c|}{$\bar{\gamma}_{e}=1$} & \multicolumn{3}{|c|}{$\bar{\gamma}_{e}=3$} & \multicolumn{3}{|c|}{$\bar{\gamma}_{e}=5$} \\
\hline & & $s_{0}=10$ & $s_{0}=20$ & $s_{0}=30$ & $s_{0}=10$ & $s_{0}=20$ & $s_{0}=30$ & $s_{0}=10$ & $s_{0}=20$ & $s_{0}=30$ \\
\hline \multirow{3}{*}{ Boat } & $P S W R_{v}^{b}=54.45$ & 1.45 & 2.86 & 4.33 & 1.24 & 2.86 & 4.33 & $\mathrm{NC}$ & 2.42 & 4.29 \\
\hline & $P S W R_{v}^{b}=51.44$ & 1.46 & 2.97 & 4.37 & 1.25 & 2.97 & 4.37 & $\mathrm{NC}$ & 2.50 & 4.35 \\
\hline & $P S W R_{v}^{b}=49.68$ & 1.46 & 2.99 & 4.50 & 1.25 & 2.99 & 4.50 & $\mathrm{NC}$ & 2.50 & 4.45 \\
\hline \multirow{3}{*}{ Goldhill } & $P S W R_{v}^{b}=54.45$ & 1.55 & 3.02 & 4.58 & 1.36 & 3.02 & 4.58 & $\mathrm{NC}$ & 2.50 & 4.53 \\
\hline & $P S W R_{v}^{b}=51.44$ & 1.56 & 3.14 & 4.68 & 1.37 & 3.14 & 4.68 & $\mathrm{NC}$ & 2.81 & 4.65 \\
\hline & $P S W R_{v}^{b}=49.68$ & 1.57 & 3.15 & 4.72 & 1.37 & 3.15 & 4.72 & $\mathrm{NC}$ & 2.81 & 4.67 \\
\hline \multirow{3}{*}{ Lena } & $P S W R_{v}^{b}=54.45$ & 1.12 & 2.17 & 3.32 & 0.62 & 2.16 & 3.32 & $\mathrm{NC}$ & 1.25 & 3.12 \\
\hline & $P S W R_{v}^{b}=51.44$ & 1.13 & 2.29 & 3.40 & 0.78 & 2.26 & 3.39 & $\mathrm{NC}$ & 1.25 & 3.28 \\
\hline & $P S W R_{v}^{b}=49.68$ & 1.13 & 2.31 & 3.47 & 0.78 & 2.26 & 3.47 & $\mathrm{NC}$ & 1.69 & 3.28 \\
\hline \multirow{3}{*}{ Tank } & $P S W R_{v}^{b}=54.45$ & 1.33 & 2.59 & 3.92 & 1.09 & 2.59 & 3.92 & $\mathrm{NC}$ & 2.17 & 3.75 \\
\hline & $P S W R_{v}^{b}=51.44$ & 1.34 & 2.71 & 4.06 & 1.11 & 2.69 & 4.06 & $\mathrm{NC}$ & 2.30 & 3.90 \\
\hline & $P S W R_{v}^{b}=49.68$ & 1.35 & 2.72 & 4.07 & 1.11 & 2.71 & 4.08 & $\mathrm{NC}$ & 2.30 & 3.98 \\
\hline
\end{tabular}

\section{Discussion}

The proposed scheme falls into the category of spread spectrum zero-bit watermark insertion based on an optimization formulation. An alternative method that falls in the exact same category is the technique for optimum watermark embedding by linear programming proposed by Pereira et al. [14]. That work adopts a similar approach, by designing an optimum watermarked image under linear constraints by employing linear programming. In the present work, we extend the class of constraints that can be employed for "embedding by optimization" from the class of linear constraints employed by Pereira et al. to a much broader class of convex constraints (since linear constraints constitute a subclass of convex constraints). In the formulation proposed here, convex and quasi-convex constraints can be incorporated for watermark embedding. Hence, among the proposed optimization based formulations, the method we propose provides the most flexible framework.

Another beneficial aspect of the proposed technique is the ease of handling constraints formulated in various transform domains. The scheme, as described, incorporates convex constraints in the spatial domain (watermark strength, watermark texture visibility), in the frequency domain (overall frequency weighted distortion), and in the spatio-DCT domain (robustness to JPEG compression). Handling these constraints that are naturally formulated in different domains is one of the main strengths of the methodology which is hard to replicate in heuristic embedding formulations. In addition the proposed scheme also allows embedding of multiple watermarks in an optimal sense while providing implicit shaping of watermark signals to meet the visual constraints. We have shown the realization of such a scheme for the feasibility formulation of the watermarking problem in our prior work [33]. The underlying advantage of meeting or optimizing perceptual distortion constraints with multiple watermarks is also applicable to the present optimization formulation, though not directly investigated here.

For the formulation of the watermark embedding strength and compression resilience constraints, the proposed methodology relies on modeling the "attacks" on the watermarked image, specifically as additive noise and JPEG compression for these two constraints. In an absolutely general setting, a modeling of all watermark attacks is not possible due to the number and complexity of the set of possible attacks. However, for a number of applications (e.g., broadcast monitoring, robust authentication in the present of network transcoding, etc.) "semi-fragile" watermarks are recognized as an acceptable, even preferable, solution. In these scenarios, the attack channel can indeed be modeled and utilized in the framework presented here. As opposed to other approaches, in these scenarios the framework presented here offers the clear advantage of allowing one critical requirement to be optimized while still meeting other constraints. In the model of watermarking as a communication problem with side information [26, Ch. 5], game theoretic models have been advantageously applied in order to model (power constrained) adversaries [26, p. 377], [34]. Similar methodologies may provide useful extensions of the framework presented here, though this is clearly beyond the scope of the present work.

Though the visual models used in the paper are relatively primitive, they capture the two main elements included in most visual system models, i.e., contrast sensitivity and texture masking. This allows us to demonstrate utility of the proposed 
optimal watermarking framework. Our framework, on the other hand, is quite general and other visual system models can be readily incorporated provided these can be formulated as, or closely approximated by, convex constraints. An investigation of more recent models [35]-[37] would be of interest as an advance over the work presented here. The principal challenge here is in formulating the visibility constraints as simple convex sets for which projections can be readily obtained.

\section{CONCLUSION}

Maximization or minimization of an objective function, subject to additional constraints, is often desirable in spread spectrum watermarking applications. Optimal watermark embedding for a number of these scenarios can be accomplished by combining a multistep feasibility formulation of the optimization [19, p.159], with set theoretic watermarking via projections onto convex sets (POCS) [17], [18]. The combination provides a general framework for determining an optimal watermarked image for convex objective functions with other convex constraints. Specifically, constraints on watermark embedding strength, compression resilience, frequency weighted perceptual distortion, and watermark texture visibility can be imposed while optimizing a metric quantifying one of these performance criteria. Experimental results demonstrate the effectiveness of the proposed methodology.

\section{APPENDIX I \\ Texture Masking Model and TeXture Visibility Metric}

We use the spatial texture masking model of Pereira et al. [14], [38]. For the formulations in Sections II-A-C we obtain pixel-wise distortion limits from this model and for the optimization of Section II-D, we formulate a texture visibility metric based on the model. We include a short synopsis of the model here and refer the reader to [14], [38] for details. The cover image is modeled as a sum of the local mean and an error term, with the latter further modeled by a generalized Gaussian distribution. A noise visibility function (NVF) at each pixel position $(i, j)$ is obtained from this model as

$$
\operatorname{NVF}(i, j)=\frac{1}{1+\kappa \sigma_{X}^{2}(i, j)}
$$

where $\sigma_{X}^{2}(i, j)$ represents the estimated local variance over a $3 \times 3$ window centered at pixel $(i, j)$ and $\kappa=\beta / \sigma_{X \text { max }}^{2}$ is a tuning parameter that controls the contrast adjustment, where $\sigma_{X \max }^{2}$ is the maximal local variance of the image and $\beta$ is an experimentally determined parameter [38].

Using the NVF the allowable pixel distortion at pixel location $(i, j)$ is computed as

$$
\Delta(i, j)=(1-\operatorname{NVF}(i, j)) s_{0}+\operatorname{NVF}(i, j) s_{1}
$$

where $s_{0}$ and $s_{1}$ represents the allowable pixel distortion in busy and flat regions, respectively. Following [38], we use $s_{0}=10 s_{1}$. The upper and lower bounds on the distortion are then set equal to $\Delta$, i.e., $D_{U}\left(X_{0}\right)=D_{L}\left(X_{0}\right)=\Delta$ utilized for the constraint in Section III. The NVF lies in $[0,1]$ and is small in regions of the cover image that are smooth, i.e., have low local variance and the NVF is large in regions of the cover image that are textured (and, therefore, have high variance). Thus, (12) indicates that a large distortion is allowed in textured regions of the cover image in comparison with the smooth regions.

From (12) we note that the smallest $s_{0}$ for which a candidate image $X$ is acceptable is given by $s_{0}^{\min }=$ $\max _{i, j}\left|X(i, j)-X_{0}(i, j)\right| /(1-0.9 \mathrm{NVF}(i, j))$. This value represents a (worst case) measure of the visibility of the texture noise $X-X_{0}$ in the candidate watermarked image $X$. Denoting $D^{*}(i, j)=1 /(1-0.9 \operatorname{NVF}(i, j))$ and $D=\operatorname{vec}\left(D^{*}\right)$, $D_{0}=\operatorname{diag}(D)$ we can represent above in compact form as: $s_{0}^{\text {min }}=\left\|D_{0}\left(X-X_{0}\right)\right\|_{\infty}$. Thus, for the formulations of Sections II-A-C the watermark texture visibility constraint can be written as $\left\|D_{0}\left(X-X_{0}\right)\right\|_{\infty} \leq s_{0}$.

\section{APPENDIX II \\ CONSTRaint Set Projection Operators}

The solution of the feasibility problem in (10) requires a computation of the projections onto the sets

$$
S_{i}\left(\alpha_{i}\right) \equiv\left\{X: \phi_{i}(X) \leq \alpha_{i}\right\} \quad i=0, \ldots, 3
$$

where $\alpha_{0}=\tau$ and the functions $\phi_{i}()$ and bounds $\left\{\alpha_{i}\right\}_{i=1}^{3}$ are as defined in Section II (following (9)).

The projection onto the set $S$ is given by $P_{S}(Y)=$ $\arg \min _{X \in S}\|Y-X\|^{2}$. The projection operators for these sets were derived in [17]. We summarize these in the following subsections.

Projection Onto $S_{0}(\alpha)$ (Embedding Strength Constraint):

$$
P_{S_{0}(\alpha)}(Y)=Y+\frac{\lambda}{2}[W-\bar{W}]
$$

where the Lagrange [39] parameter for this projection is obtained as

$$
\lambda=\left\{\begin{array}{ll}
\frac{2\left[\phi_{0}(Y)-\alpha\right]}{W^{T}(W-\bar{W})}, & \phi_{0}(Y)>\alpha \\
0, & \text { otherwise }
\end{array} .\right.
$$

Projection Onto $S_{1}(\alpha)$ (Frequency Weighted Perceptual Distortion Constraint): For shift invariant $H$, the projection and the Lagrange parameter are computed efficiently using the $2-D$ discrete Fourier Transform (DFT). Denoting the projection by $Z=P_{S_{1}(\alpha)}(Y)$, we have

$$
Z(l, k)=X_{0}(l, k)+\frac{Y(l, k)-X_{0}(l, k)}{1+\lambda|H(l, k)|^{2}}
$$

where $Z(l, k), H(l, k), Y(l, k)$ and $X_{0}(l, k)$ represent the 2-D DFT coefficients of $Z, H, Y$, and $X_{0}$, respectively, and $\lambda$ is the Lagrange parameter. For $Y \notin S_{1}(\alpha)$ the Lagrange parameter is the positive root of

$$
\frac{1}{N M} \sum_{l, k} \frac{\left|H(l, k)\left(Y(l, k)-X_{0}(l, k)\right)\right|^{2}}{\left(1+\lambda|H(l, k)|^{2}\right)^{2}}=\alpha^{2}
$$

and $\lambda$ is zero if $Y \in S_{1}(\alpha)$. 
TABLE IV

Results for Minimization of Watermark TeXture Visibility. The Table Lists the Minimum Values of the TeXture Noise Visibility Metric

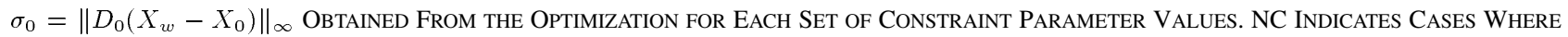
NO FEASible SOLUTION WAS Found (FOR THE CONSTRAints AlONE).

\begin{tabular}{|c|c|c|c|c|c|c|c|c|c|c|}
\hline & & \multicolumn{3}{|c|}{$\bar{\gamma}_{e}=1$} & \multicolumn{3}{|c|}{$\bar{\gamma}_{e}=3$} & \multicolumn{3}{|c|}{$\bar{\gamma}_{e}=5$} \\
\hline & & $\bar{\gamma}_{c}=1$ & $\bar{\gamma}_{c}=2$ & $\bar{\gamma}_{c}=3$ & $\bar{\gamma}_{c}=1$ & $\bar{\gamma}_{c}=2$ & $\bar{\gamma}_{c}=3$ & $\bar{\gamma}_{c}=1$ & $\bar{\gamma}_{c}=2$ & $\bar{\gamma}_{c}=3$ \\
\hline \multirow{3}{*}{ Boat } & $P S W R_{v}^{b}=54.45$ & 6.99 & 14.10 & 21.28 & 9.17 & 14.49 & 21.28 & 15.50 & 17.61 & 22.53 \\
\hline & $P S W R_{v}^{b}=51.44$ & 6.99 & 13.55 & 20.19 & 8.94 & 13.94 & 20.19 & 14.96 & 17.53 & 21.91 \\
\hline & $P S W R_{v}^{b}=49.68$ & 6.99 & 13.47 & 20.11 & 8.94 & 13.86 & 20.11 & 14.96 & 17.14 & 21.75 \\
\hline \multirow{3}{*}{ Goldhill } & $P S W R_{v}^{b}=54.45$ & 6.52 & 12.85 & 19.72 & 8.78 & 13.24 & 19.72 & 15.11 & 16.91 & 21.36 \\
\hline & $P S W R_{v}^{b}=51.44$ & 6.44 & 12.77 & 19.10 & 8.47 & 13.16 & 19.10 & 14.49 & 16.75 & 21.28 \\
\hline & $P S W R_{v}^{b}=49.68$ & 6.44 & 12.77 & 19.02 & 8.47 & 13.16 & 19.02 & 14.49 & 16.44 & 20.82 \\
\hline \multirow{3}{*}{ Lena } & $P S W R_{v}^{b}=54.45$ & 8.94 & 18.32 & 27.22 & 11.44 & 18.47 & 27.22 & 18.16 & 22.14 & 28.78 \\
\hline & $P S W R_{v}^{b}=51.44$ & 8.86 & 17.46 & 26.13 & 11.13 & 17.93 & 26.13 & 17.77 & 21.91 & 28.47 \\
\hline & $P S W R_{v}^{b}=49.68$ & 8.86 & 17.38 & 25.97 & 11.13 & 17.85 & 25.97 & 17.46 & 21.60 & 27.85 \\
\hline \multirow{3}{*}{ Tank } & $P S W R_{v}^{b}=54.45$ & 7.53 & 15.66 & 22.53 & 9.72 & 15.74 & 22.53 & 15.50 & 20.03 & 24.49 \\
\hline & $P S W R_{v}^{b}=51.44$ & 7.46 & 14.80 & 22.14 & 9.49 & 15.19 & 22.14 & 14.96 & 18.78 & 23.86 \\
\hline & $P S W R_{v}^{b}=49.68$ & 7.46 & 14.72 & 22.07 & 9.49 & 15.11 & 22.07 & 14.96 & 18.39 & 23.78 \\
\hline
\end{tabular}

Projection Onto $S_{2}(\alpha)$ (Watermark Texture Visibility Constraint): The constraint set $S_{2}(\alpha)$ actually represents a collection of $M N$ term-wise upper and lower bounds on the $M N$ entries in $X$. Using the notation of Appendix I these $M N$ constraints can be written as

$$
L_{j} \leq X_{j} \leq U_{j}, \quad j=1,2 \ldots, M N
$$

where $L=X_{0}-D_{L}\left(X_{0}\right), U=X_{0}+D_{U}\left(X_{0}\right)$ and $V_{j}$ denotes the $j^{\text {th }}$ element of the vector $V$. The projection $Z=P_{S_{2}(\alpha)}(Y)$ is then obtained term-by-term as

$$
Z_{j}=\left\{\begin{array}{ll}
Y_{j} & \text { if } L_{j} \leq Y_{j} \leq U_{j} \\
L_{j} & \text { if } Y_{j}<L_{j} \\
U_{j} & \text { if } Y_{j}>U_{j}
\end{array} .\right.
$$
straint):

Projection Onto $S_{3}(\alpha)$ (Robustness to Compression Con-

$$
P_{S_{3}(\alpha)}(Y)=Y+\frac{\lambda}{2}\left[\mathcal{T}_{\mathcal{C}}^{T} W-\overline{\mathcal{T}_{\mathcal{C}}^{T} W}\right]
$$

where $\lambda$ is the Lagrange parameter given by

$$
\lambda= \begin{cases}\frac{2\left[\phi_{3}(Y)-\alpha\right]}{W^{T} \mathcal{T}_{\mathcal{C}}\left(\mathcal{T}_{\mathcal{C}}{ }^{T} W-\overline{\mathcal{T}_{\mathcal{C}}}{ }^{T} W\right.}, & \phi_{3}(Y)>\alpha \\ 0, & \text { otherwise }\end{cases}
$$

\section{ACKNOWLEDGMENT}

The authors would like to thank the anonymous reviewers for their comments that have improved the paper.

\section{REFERENCES}

[1] O. Altun, G. Sharma, and M. Bocko, "Optimum watermark design by vector space projections," in Proc. Int. Conf. Image Processing, 2006, pp. 1413-1416.

[2] I. Cox, M. Miller, J. Bloom, J. Fridrich, and T. Kalker, Digital Watermarking and Steganography, 2nd ed. San Francisco, CA: Morgan Kaufmann, 2007.

[3] C. Podilchuk and E. Delp, "Digital watermarking: algorithms and applications," IEEE Signal Process. Mag., vol. 18, no. 4, pp. 33-46, Jul. 2001.

[4] G. Langelaar, I. Setyawan, and R. Lagendijk, "Watermarking digital image and video data," IEEE Signal Process. Mag., vol. 17, no. 5, pp. 20-46, Sep. 2000.

[5] M. Barni and F. Bartolini, "Data hiding for fighting piracy," IEEE Signal Process. Mag., vol. 21, no. 2, pp. 28-29, Mar. 2004.

[6] M. U. Celik, G. Sharma, and A. M. Tekalp, "Lossless watermarking for image authentication: A new framework and an implementation," IEEE Trans. Image Process., vol. 15, no. 4, pp. 1042-1049, Apr. 2006.

[7] T. Ohsawa and M. Karita, "Automatic Telecasting or Radio Broadcasting Monitoring System," U.S. patent (3,760,275), 1973.

[8] M. Wu, W. Trappe, Z. Wang, and K. Liu, "Collusion-resistant fingerprinting for multimedia," IEEE Signal Process. Mag., vol. 21, no. 2, pp. 15-27, Feb. 2004

[9] I. Cox, J. Kilian, F. T. Leighton, and T. Shamoon, "Secure spread spectrum watermarking for multimedia," IEEE Trans. Image Process., vol. 6, no. 12, pp. 1673-1687, Dec. 1997.

[10] K. Solanki, N. Jacobsen, U. Madhow, B. S. Manjunath, and S. Chandrasekaran, "Robust image-adaptive data hiding based on erasure and error correction," IEEE Trans. Image Process., vol. 13, no. 12, pp. 1627-1639, Dec. 2004

[11] M. L. Miller, G. J. Doërr, and I. J. Cox, "Dirty-paper trellis codes for watermarking," in Proc. Int. Conf. Image Processing, 2002, pp. 129-132.

[12] J. Fridrich, M. Goljan, and D. Soukal, "Wet paper codes with improved embedding efficiency," IEEE Trans. Inf. Forensics Security, vol. 1, no. 1, pp. 102-110, Jan. 2006

[13] B. Chen and G. W. Wornell, "Quantization index modulation: A class of provably good methods for digital watermarking and information embedding," IEEE Trans. Inf. Theory, vol. 47, no. 4, pp. 1423-1443, May 2001. 
[14] S. Pereira, S. Voloshynoskiy, and T. Pun, "Optimal transform domain watermark embedding via linear programming," Signal Process., vol. 81, no. 6, pp. 1251-1260, Jun. 2001.

[15] M. K. Mihcak, R. Venkatesan, and M. Kesal, "Optimization algorithms for quantizing randomized statistics of image regions," presented at the 40th Annu. Allerton Conf. Communication, Control and Computing, Oct. 2002.

[16] M. L. Miller, I. J. Cox, and J. A. Bloom, "Informed embedding: Exploiting image and detector information during watermark insertion," presented at the Int. Conf. Image Processing, 2000.

[17] O. Altun, G. Sharma, M. Celik, and M. Bocko, "A set theoretic framework for watermarking and its application to semifragile tamper detection," IEEE Trans. Inf. Forensics Security, vol. 1, no. 4, pp. 479-492, Dec. 2006.

[18] O. Altun, G. Sharma, M. Celik, and M. Bocko, "Semifragile hierarchical watermarking in a set theoretic framework," in Proc. Int. Conf. Image Processing, 2005, vol. 1, pp. 1001-1004.

[19] S. Boyd, Convex Optimization. Cambridge, U.K.: Cambridge Univ. Press.

[20] H. Stark and Y. Yang, Vector Space Projections: A Numerical Approach to Signal and Image Processing, Neural Nets, and Optics. New York: Wiley, 1998.

[21] H. J. Trussell and M. R. Civanlar, "The feasible solution in signal restoration," IEEE Trans. Acoust. Speech Signal Process., vol. ASSP-32, no. 4, pp. 201-212, Apr. 1984.

[22] P. L. Combettes, "The foundations of set theoretic estimation," Proc. IEEE, vol. 81, no. 2, pp. 182-208, Feb. 1993.

[23] Y. Yang, N. P. Galatsanos, and A. Katsaggelos, "Projection-based spatially adaptive reconstruction of block transform compressed images," IEEE Trans. Image Process., vol. 4, no. 7, pp. 896-908, Jul. 1995.

[24] G. Sharma, "Set theoretic estimation for problems in subtractive color," Color Res. Appl., vol. 25, no. 4, pp. 333-348, Oct. 2000.

[25] G. Sharma and H. J. Trussell, "Set theoretic signal restoration using an error in variables criterion," IEEE Trans. Image Process., vol. 6, no. 12, pp. 1692-1697, Dec. 1997.

[26] I. J. Cox, M. L. Miller, and J. A. Bloom, Digital Watermarking. San Francisco, CA: Morgan Kaufmann, 2001.

[27] J. L. Mannos and D. L. Sakrison, "The effects of a visual fidelity criterion on the encoding of images," IEEE Trans. Inf. Theory, vol. 20, no. 7, pp. 525-536, Jul. 1974.

[28] V. K. Goyal, "Theoretical foundations of transform coding," IEEE Signal Process. Mag., vol. 18, no. 9, pp. 9-21, Sep. 2001.

[29] S. Pereira, S. Voloshynoskiy, and T. Pun, "Optimal transform domain watermark embedding via linear programming," Signal Process., vol. 81 , no. 6, pp. 1251-1260, 2001.

[30] L. M. Bregman, "The method of successive projection for finding a common point of convex sets," Dokl. Akad. Nauk. SSSR, vol. 162, no. 3, pp. 688-692, 1965.

[31] P. L. Combettes, "The foundations of set theoretic estimation," Proc. IEEE, vol. 81, no. 1, pp. 182-208, Feb. 1993.

[32] [Online]. Available: http://sipi.usc.edu/database/

[33] O. Altun, G. Sharma, and M. Bocko, C. A. Bouman and E. L. Miller, Eds., "Multiple watermarking: A vector space projections approach," in Proc. SPIE: Computational Imaging IV, Jan. 2006, vol. 6065, pp. 60650O1-60650O12.

[34] P. Moulin and J. A. O'Sullivan, "Information-theoretic analysis of information hiding," IEEE Trans. Inf. Theory, vol. 49, no. 3, pp. 563-593, Mar. 2003.

[35] A. B. Watson and J. A. Solomon, "Model of visual contrast gain control and pattern masking," J. Opt. Soc. Amer., vol. 14, no. 19, pp. 2379-2391, 1994.

[36] J. Lubin, "A visual discrimination model for imaging system design and evaluation," in Vision Models for Target Detection and Recognition, E. Peli, Ed. Singapore: World Scientific, 1995, pp. 245-283.

[37] S. Daly, "A visual model for optimizing the design of image processing algorithms," in Proc. IEEE Int. Conf. Image Processing, Austin, TX, Nov. 1994, vol. 2, pp. 16-20.

[38] S. Voloshynoskiy, A. Herrigel, N. Baumgaertner, and T. Pun, "A stochastic approach to content adaptive digital image watermarking," in Proc. 3rd Int. Workshop on Information Hiding, Dresden, Germany, 1999, pp. 211-236.

[39] D. G. Luenberger, Linear and Nonlinear Programming. Reading, MA: Addison Wesley, 1989.

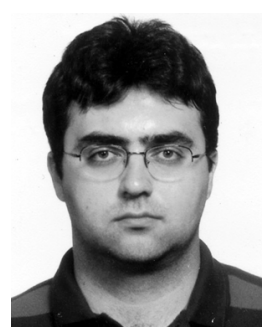

H. Oktay Altun (S'03) received the B.Sc. degree in electrical and electronic engineering in 2000 from Bilkent University, Ankara, Turkey, and the M.Sc. degree in electrical and computer engineering from the University of Rochester, Rochester, NY, in 2005.

Since January 2003, he has been a Research Assistant at the Electrical and Computer Engineering Department, University of Rochester. His research interests include multimedia security, digital watermarking, image processing, and analog circuit optimization.He is the recipient of best student paper award at WNYIPW 2005 and best poster award at CSAW 2007.

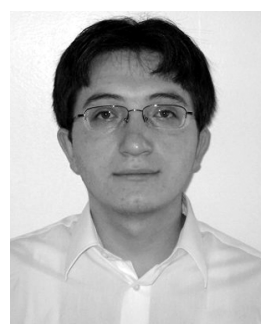

Adem Orsdemir ( $\mathrm{S}^{\prime} 07$ ) received the B.Sc. degree in electrical and electronic engineering from Bilkent University, Ankara, Turkey, in 2006, and the M.Sc. degree in electrical and computer engineering from the University of Rochester, Rochester, NY, in 2008.

Currently, he is a Research Assistant with the Electrical and Computer Engineering Department, University of Rochester. His research interests include multimedia security, digital watermarking, and image processing.

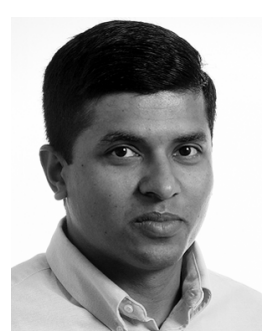

Gaurav Sharma (SM'00) received the B.E. degree in electronics and communication engineering from the Indian Institute of Technology Roorkee (formerly University of Roorkee), India, in 1990, the M.E. degree in electrical communication engineering from the Indian Institute of Science, Bangalore, India, in 1992, and the M.S. degree in applied mathematics and the Ph.D. degree in electrical and computer engineering from North Carolina State University (NCSU), Raleigh, in 1995 and 1996, respectively.

From August 1992 through August 1996, he was a Research Assistant at the Center for Advanced Computing and Communications, Electrical and Computer Engineering Department, NCSU. From August 1996 through August 2003, he was with Xerox Research and Technology, Webster, NY, initially as a member of research staff and subsequently at the position of principal scientist. Since Fall 2003, he has been an Assosociate Professor at the University of Rochester, Rochester, NY, in the Department of Electrical and Computer Engineering and the Department of Biostatistics and Computational Biology. His research interests include multimedia security and watermarking, color science and imaging, genomic signal processing, and image processing for visual sensor networks. He is the editor of the Color Imaging Handbook (CRC, 2003).

Dr. Sharma is a member of Sigma Xi, Phi Kappa Phi, Pi Mu Epsilon, IS\&T, and the signal processing and communications societies of the IEEE. He was the 2007 chair for the Rochester section of the IEEE and served as the 2003 chair for the Rochester chapter of the IEEE signal processing society. He currently serves as the vice-chair for the IEEE Signal Processing Society's Image and Multidimensional Signal Processing (IMDSP) Technical Committee and is a member of the IEEE Standing Committee on Industry DSP. He currently serves as an Associate Editor for IEEE TRANSACTIONS ON IMAGE PROCESSING, the IEEE TRANSACTIONS ON INFORMATION FORENSICS AND SECURITY, and the Journal of Electronic Imaging.

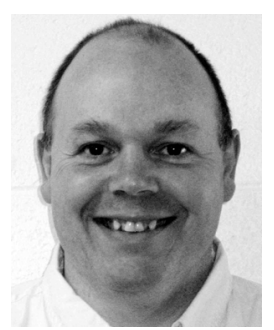

Mark F. Bocko (M'94) received the B.A. degree in physics and astronomy from Colgate University, Hamilton, NY, in 1978, and the Ph.D. degree in physics from the University of Rochester, Rochester, NY, in 1984.

He has been on the faculty of the Electrical and Computer Engineering Department, University of Rochester, since 1985, where he is now a Full Professor and Chairman of the Department. His research interests span a number of areas including sensors and integrated sensor systems, audio and music signal processing, precision measurements, superconducting electronics quantum noise, and quantum computing. 